FSU-SCRI-95-101

Oct 1995

\title{
The Phase Structure of the Schwinger Model on the Lattice with Wilson Fermions in the Hartree-Fock Approximation
}

\author{
Ivan Horváth \\ Supercomputer Computations Research Institute \\ Florida State University \\ Tallahassee, FL 32306-4052
}

\begin{abstract}
The phase diagram of the Schwinger model on the lattice with one and two degenerate flavours of Wilson fermions is investigated in the Hartree-Fock approximation. In case of a single flavour (not directly amenable to numerical simulation), the calculation indicates the existence of the parity violating phase at both weak and intermediate-to-strong couplings. In the broken phase, the Hartree-Fock vacuum sustains a nonzero electric field. With two flavours, parity is not broken at weak coupling. However, both parity and flavour become spontaneously broken at the Hartree-Fock level as the coupling becomes strong.
\end{abstract}




\section{Introduction}

Understanding lattice fermions has been an outstanding issue since the beginnings of lattice field theory but a satisfactory insight is still missing. Aside from nontrivial numerical complications introduced by fermionic degrees of freedom, there are well-known conceptual obstacles, usually referred to as the "fermion doubling problem". As revealed by the Nielsen-Ninomiya theorem [1], the doubling problem is intimately connected to chiral symmetry. In fact, this "No-Go" theorem has succeeded so far in preventing us from formulating chiral gauge theory on the lattice, although the investigations have intensified recently (see e.g. [2,3] and references therein) and possible clues might be at hand.

Except from fundamental importance of chiral symmetry in electroweak theory, chiral symmetry has long been believed crucial in understanding the low energy behaviour of strong interactions, described by a vectorlike theory like QCD. Hinted by the small pion masses, the basic starting point is that the approximate chiral symmetry crucially shapes the way the low energy strong interacting world looks. Pions are regarded as Goldstone bosons coming from the spontaneous breakdown of this chiral symmetry, and the powerful predictions of the current algebra follow [4].

It is desirable to study these interesting issues within the nonperturbative framework of lattice QCD. Nielsen-Ninomiya theorem doesn't directly prevent us from "latticizing" a vectorlike theory, even in the chiral limit. However, the existence of the chiral anomaly complicates the situation considerably. The standard argument is that since the lattice is a physical regulator, any symmetry of the lattice action will remain the valid symmetry of the theory at every stage, including the continuum limit. Consequently, an explicitly chirally symmetric lattice model can not reproduce the anomaly structure of the vectorlike theory. Indeed, the most extensively used versions of lattice QCD, namely with Wilson fermions and Kogut-Susskind fermions, both explicitly violate chiral symmetry. Nevertheless, it can be shown in lattice perturbation theory that the amount of violation is just right to obtain the correct anomaly in the continuum limit [5]. This is of course quite comforting. However, to understand chiral symmetry on the lattice at finite lattice spacing (where all the numerical simulations are performed) means to relate the concepts of chiral symmetry 
breaking to those of the lattice system, where chiral symmetry is not present.

Restricting myself now to the case of Wilson fermions, it is generally believed that there is a line of phase transitions $\kappa_{c}(g)$ (in the hopping parameter - gauge coupling plane) running up from the QCD fixed point $\kappa_{c}(0)$. On this line, pion-like state becomes massless and the continuum chiral QCD is believed to be approached by following this line towards $\kappa_{c}(0)$. Some time ago Aoki [6] set out to answer the questions raised in the previous paragraph in this context. Namely, since at $g \neq 0$ the masslessness of the pion can't be due to the spontaneous chiral symmetry breaking, what is it due to? In other words, what is the nature of the phase transition along $\kappa_{c}(g)$ ?

Aoki's answer was that $\kappa_{c}(g)$ represents the line of phase transitions at which parity (one-flavour case) or parity and flavour (multi-flavour case) becomes spontaneously broken. For one flavour, the pion is identified with the massless particle driving the parity violating phase transition $\left(\left\langle i \bar{\psi} \gamma_{5} \psi\right\rangle \neq 0\right)$. In case of two flavours, it is $\left\langle i \bar{\psi} \gamma_{5} \tau_{3} \psi\right\rangle$ acquiring an expectation value. $\pi^{0}$ is identified with a massless mode of this phase transition, while the charged pions are viewed as the Goldstone bosons coming from the breakdown of flavour. $\left\langle\bar{\psi} \gamma_{5} 1 \psi\right\rangle$ is always assumed to be zero thus giving no reason for $\eta$ to be light, which could be viewed as a solution of the $\mathrm{U}(1)$ problem on a lattice [6].

At strong coupling, the supporting evidence for this scenario is quite convincing [6]. On the other hand in the weak coupling regime (relevant for the approach to the continuum), the situation is far from conclusive. Despite the fact that some numerical work has been [7] and continues to be done [8], the very existence of the parity-flavour violating phase still needs to be examined, let alone the detailed picture of symmetry breaking. The existence of the parity-flavour violating phase has been recently established in the Nambu-JonaLasinio model using large $\mathrm{N}$ methods [9] and also in a numerical simulations with finite number of colours $(\mathrm{N}=2)$ [10]. In that case, the parity-flavour breaking phase in a model with two flavours seems only to exist at strong and intermediate couplings.

Recently, there has been a line of seemingly unrelated developments taking place concerning chiral symmetry following the ideas of Kaplan [11]. His approach amounts to the use of the surface modes of the vectorlike theory with Wilson fermions in $2 d+1$ dimensions as a basis for the construction of $2 d$-dimensional chiral gauge theory on the 
lattice. (For review, see [12] and references therein.) Detailed Hamiltonian analysis of the surface modes of Wilson fermions has been carried out in [13], where it was also suggested that the notion of surface modes might be useful for understanding the conventional Wilson formulation as well. In particular, one can think of the appearance of the surface modes as an underlying mechanism generating the parity violating phase.

The nature of the argument is as follows. Consider the Hamiltonian for one flavour of free Wilson fermions in one spatial dimension on a finite lattice with open boundaries

$$
H_{W}=K \sum_{j}\left[\bar{\psi}_{j+1}\left(i \gamma_{1}-r\right) \psi_{j}-\bar{\psi}_{j}\left(i \gamma_{1}+r\right) \psi_{j+1}\right]+M \sum_{j} \bar{\psi}_{j} \psi_{j}
$$

Here $K, M, r$ are hopping parameter, mass and the Wilson parameter respectively. $\psi_{j}$ is a two component spinor living on site $j$. If one increases the hopping parameter (or equivalently decreases the mass) to the supercrticilal values, so that

$$
\left|\frac{M}{2 K r}\right|<1
$$

two levels start to behave differently from the rest of the spectrum and appear bound to the ends of the lattice [13]. As the size L of the system goes to infinity, the energy of these surface modes tends to zero. On a finite lattice the two modes mix and acquire the energy $\epsilon \sim e^{-L}$. Consider now the Dirac vacuum with all the negative energy levels filled. The last filled level will either be the surface mode on the right or the one on the left *, thus creating an asymmetric distribution of particles in the vacuum with an extra particle on one end. Consequently, after turning on the $U(1)$ gauge field, this will generate an electric field running through the vacuum. In one spatial domension such a field can not be canceled by a pair production and we find that parity is not respected by the vacuum of this theory.

These ideas were further developed in a recent inspirative review by Creutz [2]. He gives a comprehensive qualitative picture of the phase structure using the surface modes scenario in both single and multi-flavour case. The argument is based on the frequently

\footnotetext{
* On a finite lattice I assume that some very small left-right symmetry breaking term is added to the $H_{W}$, so that the left and right modes do not mix.
} 
used analogy between nonabelian gauge theories in four dimensions and electrodynamics in two dimensions $\left(Q E D_{2}\right.$, massive Schwinger model). As is well known, $Q E D_{2}$ exhibits some of the most intriguing features ascribed to QCD, namely confinement, chiral symmetry breaking and the existence of the $\theta$ parameter. As such it represents a popular toy model for QCD with the advantage that it can be analysed semiclassically through bosonisation [14]. Taking lessons from the continuum and combining them with the existence of the doublers and surface modes on the lattice, one naturally arrives to the conclusion that the physics of the parity violating phase corresponds to the $\theta=\pi$ case in the continuum. This is based on the considerations of the previous paragraph and the fact that in $Q E D_{2}$ the $\theta$ parameter has a direct physical meaning as the background electric field. The phase diagrams of Ref. [2] represent the expected positions of $\theta=\pi$ transitions on the lattice that should be applicable at weak coupling.

The purpose of this paper is to investigate the issues of a parity violating phase in lattice $Q E D_{2}$ with Wilson fermions in a direct lattice calculation. Compared to the wealth of exact and approximate information accumulated over the years on the continuum Schwinger model $*$, the knowledge we have on the lattice is rather modest. First of all, there are no exact solutions within any of the formulations where doublers are removed. The model was mostly investigated with Kogut-Susskind fermions, testing various numerical methods by comparing the lattice results to the exactly known continuum quantities in the massless case (see e.g. [16]). However, very little is known about the theory in the Wilson fermion formulation. The situation is particularly interesting for a single flavour, because in that case the direct numerical simulation is not possible. This is due to the fact that for certain gauge configurations the fermionic determinant is not positive and therefore one does not have a probabilistic weight for the purposes of Monte Carlo simulation. In a recent work [17], Gausterer and Lang studied the Lee Yang zeros of the partition function on small lattices analytically (at infinite coupling) and numerically (at intermediate couplings). The system was also studied in Ref. [18]. In case of two flavours, the direct numerical analysis is possible, but to my knowledge, the systematic study of phase structure has not been carried out.

\footnotetext{
* The recent activity concentrated mostly on the multi-flavour case. See e.g. [15].
} 
In what follows, I will study the phase diagram of Hamiltonian $Q E D_{2}$ on the lattice with one and two degenerate flavours of Wilson fermions in the Hartree-Fock (H-F) approximation. I will work in the axial gauge, where the gauge degrees of freedom are easily eliminated in favour of fermionic fields, thus providing a convenient setup for the use of the H-F approximation to the vacuum of the theory. The H-F ground state, or the independent fermion ground state, is a state one might call (by definition) the mean field ground state for the theory of interacting, particle number conserving (charge conserving) fermions. It has a nice variational interpretation and can be regarded as a first term in a series of systematic variational improvements. While belonging to the standard set of techniques used in many-body theory, H-F methods are rarely invoked in the lattice gauge context. In the case under consideration, however, it can give us valuable hints about the phase structure of the theory.

Investigating the phase diagram, I adopt the point of view, taken in Ref. [2], that it is quite natural to introduce the axial mass term (the " $M_{5}$ "-term). More specifically, I will consider the generalized version of Hamiltonian (1), given by

$$
H_{W_{5}}=H_{W}+M_{5} i \bar{\psi}_{j} \gamma_{5} \psi_{j} \equiv H_{W}+H_{5} .
$$

Indeed, one obtains such a term from the conventional mass term by a chiral rotation. The existence of a $\theta$ parameter in the continuum theory can be thought of as being due to the fact that because of regularization, the theory with such a rotated mass term is actually not equivalent to the original one and is thus anomalous. In this way, introducing $M_{5}$ essentially means trading $\theta$ (being an independent parameter of the theory) in favour of this new mass term. Consequently, I will consider the lattice theory in the space of three bare parameters, $M, M_{5}$ and $g$ (gauge coupling), in contrast to three conventional continuum parameters $m, \theta$ and $e$.

In Sec. II, starting from the continuum theory in axial gauge, I will formulate the lattice model with one flavour and discuss its discrete symmetries. The H-F approximation is then described in Sec. III. I do this in some detail, stressing the variational character of the method and the fact that all the discrete symmetries (including parity) are preserved by the approximation. The numerical solutions of the H-F equations on finite lattices and 
their implications on the H-F phase diagram are discussed in Sec. IV. I try to make all the

observed qualitative features of the vacuum plausible by tracing them to the elementary picture of interacting particles in filled H-F levels. The model with two flavours is then formulated and analysed in Secs. V and VI. Summary, together with some generalizations and speculations, is given in Sec. VII. Finally, the numerical procedure used to solve the $\mathrm{H}-\mathrm{F}$ equations is described in Appendix.

\section{The Model with One Flavour}

The massive Schwinger model with one flavour of fermions is defined by the Lagrangian

$$
\mathcal{L}=-\frac{1}{4} F_{\mu \nu} F^{\mu \nu}+\bar{\psi}\left[\gamma^{\mu}\left(i \partial_{\mu}-e A_{\mu}\right)-m\right] \psi
$$

where $F_{\mu \nu}=\partial_{\mu} A_{\nu}-\partial_{\nu} A_{\mu}$. $\psi$ is the two-component spinor field, $A_{\mu}$ is the gauge field and $m$, e the mass and dimensionful coupling constant respectively. I will consider the theory in axial gauge $\left(A_{1}=0\right)$.

The equation of motion for $A_{0}$ is the equation of constraint (Gauss' law)

$$
E^{\prime}(x) \equiv-A_{0}^{\prime \prime}(x)=e \psi^{\dagger}(x) \psi(x) \equiv e \rho(x)
$$

where $E(x) \equiv-A_{0}^{\prime}(x)$ is the electric field and $\rho$ the charge density. It can be solved by

$$
A_{0}(x)=-\frac{1}{2} \int d x^{\prime}\left|x-x^{\prime}\right| e \rho\left(x^{\prime}\right)+B x+C .
$$

The integration constant $C$ is physically irrelevant and will be put to zero. The constant $B$ represents a uniform background field which can be also put to zero for my current purposes. Then the Hamiltonian of the theory for the zero total charge takes the form

$$
H=\int d x\left[\bar{\psi}\left(i \gamma_{1} \partial_{1}+m\right) \psi-\frac{e^{2}}{4} \int d x^{\prime} \rho(x)\left|x-x^{\prime}\right| \rho\left(x^{\prime}\right)\right] .
$$

Upon quantization, $\psi$ and $H$ become operators in the corresponding Hilbert space, with field operators subject to the canonical anticommutation relations. In the standard treatment, the local charge density operator is replaced by its normal ordered version 
$: \psi^{\dagger} \psi$ : with the normal ordering usually performed with respect to the filled Dirac sea (no particles, no antiparticles). This effectively amounts to the compensation of the infinite charge, generated by the sea. Since I will not introduce the antiparticle operators it is more convenient for my later purposes to define the charge density in such a way that the compensation is explicit, namely

$$
\rho(x) \equiv \psi^{\dagger}(x) \psi(x)-1
$$

To formulate this theory on a lattice is now straightforward. It will be defined by the Hamiltonian

$$
H=H_{W_{5}}-\frac{g^{2}}{4} \sum_{n, m} \rho_{n}|n-m| \rho_{m} \equiv H_{W_{5}}+H_{I},
$$

where $H_{W_{5}}$ is the free part with $H_{5}$ defined in (3) and

$$
\rho_{n}=\psi_{n}^{\dagger} \psi_{n}-1
$$

The indices $n, m$ label the lattice sites runing from 1 to $L$ and the lattice spacing has been set to unity. Fermionic variables are subject to open boundary conditions and satisfy the canonical anticommutation relations

$$
\left\{\psi_{n}^{\sigma}, \psi_{m}^{\dagger \tau}\right\}=\delta_{n m} \delta_{\sigma \tau}
$$

with $\sigma, \tau$ being the spinor indices. The electric field in this formulation is a derived quantity, defined through the the lattice analog of Gauss' law (5) by

$$
E_{j}=\frac{g}{2}\left[\sum_{l=1}^{j} \rho_{l}-\sum_{l=j+1}^{L} \rho_{l}\right] .
$$

Here $E_{j}$ is the operator of electric field on link $(j, j+1)$.

It is worth emphasizing at this point that similarly to the continuum case, the lattice interacting theory posesses exact discrete symmetries. Choosing the representation of $\gamma$ matrices as

$$
\gamma^{0}=\left(\begin{array}{cc}
0 & 1 \\
1 & 0
\end{array}\right) \quad \gamma^{1}=\left(\begin{array}{cc}
0 & 1 \\
-1 & 0
\end{array}\right) \quad \gamma_{5}=\gamma^{0} \gamma^{1}
$$


the operations of $\mathrm{C}, \mathrm{P}, \mathrm{T}$ in continuum (left column) and on the lattice (right column) are defined by the following transformation properties of the field operators

$$
\begin{array}{ll}
P \psi(x) P^{-1}=\gamma_{0} \psi(-x) & P \psi_{j} P^{-1}=\gamma_{0} \psi_{L+1-j} \\
C \psi(x) C^{-1}=\gamma_{1} \bar{\psi}^{T}(x) & C \psi_{j} C^{-1}=\gamma_{1} \bar{\psi}_{j}^{T} \\
T \psi(x) T^{-1}=\gamma_{0} \psi(x) & T \psi_{j} T^{-1}=\gamma_{0} \psi_{j} .
\end{array}
$$

Here $C, P$ are unitary and $T$ antiunitary operators. The above transformations indeed leave the corresponding continuum and lattice Hamiltonians without $H_{5}\left(M_{5}=0\right)$ unchanged.

Note that $H_{5}$ is odd under $P, C$ and even under $T$. Therefore $C P$ and $T$ are the exact symmetries of the theory at any $M_{5}$. This is different from the situation in four dimensions, where $H_{5}$ is invariant under $C$ and changes sign under $P, T$, which in turn implies that both $P C$ and $T$ are explicitly broken by nonzero $M_{5}$.

\section{Hartree-Fock Approximation}

In solid state physics, the H-F approximation is frequently referred to as the "independent electron approximation" and this probably captures its essence best. Indeed, the main idea is to approximate a given state of the fermionic many-body system by a Slater determinant of some set of one-particle states. This is usually applied to approximate the unknown vacuum of the theory, which is also my main interest here. In that case the method boils down to finding a set of one-particle states with the Slater determinant of minimal energy.

Let me therefore start to investigate the vacuum of the one-flavour lattice Schwinger model by considering an arbitrary (but fixed) complete orthonormal set of one-particle fermionic states on a lattice of $L$ sites

$$
S \equiv\left\{\phi^{\alpha} \mid \alpha=1,2, \ldots, 2 L\right\}
$$

Every state $\phi^{\alpha}$ is a collection of two-component spinors, residing on site $n$

$$
\phi^{\alpha} \equiv\left\{\phi_{n}^{\alpha} \mid n=1,2, \ldots, L\right\} \quad \phi_{n}^{\alpha} \equiv\left(\begin{array}{c}
\phi_{n}^{\alpha, 1} \\
\phi_{n}^{\alpha, 2}
\end{array}\right) .
$$


One can build the fermionic many-body Fock space out of these states in a standard way and define the complete set of fermionic annihilation operators by

$$
\psi_{n}=\sum_{\alpha} a_{\alpha} \phi_{n}^{\alpha}
$$

With definition (10) of the local charge operator, restriction to the charge zero sector translates into the requirement of half-filling. In other words, only states with $L$ particles are allowed. An arbitrary state $|\psi\rangle$ from this subspace can be written in the form

$$
|\psi\rangle=c^{0}|0 f, 0 \bar{f}\rangle+\sum_{r} c_{r}^{1}|1 f, 1 \bar{f}\rangle_{r}+\sum_{r} c_{r}^{2}|2 f, 2 \bar{f}\rangle_{r}+\ldots+c^{L}|L f, L \bar{f}\rangle .
$$

Here $|0 f, 0 \bar{f}\rangle$, the "sea", is the state with first $L$ levels filled

$$
|0 f, 0 \bar{f}\rangle=a_{1}^{\dagger} a_{2}^{\dagger} \ldots a_{L}^{\dagger}|0\rangle
$$

$|n f, n \bar{f}\rangle$ generically represents states with $n$ fermions removed from the sea and put into $n$ empty levels ( $n$ fermion - $n$ antifermion states). Index $r$ enumerates these basis states at fixed $n$. Obviously, the dimension of the charge zero sector is $\left(\begin{array}{c}2 L \\ L\end{array}\right)$.

Apart from charge conservation, no other symmetries are assumed to be respected by the vacuum. One usually restricts the space of states further by going to subspace of zero momentum. However, momentum is not well defined on a lattice with open boundaries. Specification of $\left(\begin{array}{c}2 L \\ L\end{array}\right)$ complex coefficients $c_{r}^{k}$ in the above decomposition therefore constitutes an exact representation of the vacuum. Requirement of minimal energy defines a variational problem for determination of these coefficients. However, on a reasonably sized lattices the number of variables in the problem becomes too huge to be manageable.

The variational philosophy behind the H-F approach is to regard the set $S$ as a collection of variational parameters instead. In particular, the method aims at adjusting the one-particle basis in such a way that while retaining only the first term in decomposition (18), the lowest energy state is achieved. Note that in this way, the variational problem involving $2\left(\begin{array}{c}2 L \\ L\end{array}\right)$ real variables is replaced by one involving $3 L^{2}$ real variables *. Moreover,

* Counting here includes the fact that only the filled levels represent the true variational variables since only they contribute to the total energy. One also has to take into account the orthonormality constraints. 
fixing $S$ by the Hartree-Fock prescription transforms the decomposition (18) into a well defined variational improvement scheme. Indeed, employing the H-F basis one expects the states $|n f, n \bar{f}\rangle$ to play increasingly less important roles in the true vacuum with increasing $n$. This is expected to be true regardless of the value of the gauge coupling.

Having defined the H-F approximation it is now a straightforward matter to transform the problem into the familiar manageable form. Using the field decomposition (17) one can rewrite the free part of the Hamiltonian (9) as

$$
H_{W_{5}} \equiv \sum_{n m} \psi_{n}^{\dagger} K_{n m} \psi_{m}=\sum_{\alpha \beta} a_{\alpha}^{\dagger} \mathcal{K}^{\alpha \beta} a_{\beta}
$$

where

$$
\begin{aligned}
\mathcal{K}^{\alpha \beta} & =\sum_{n m} \phi_{n}^{\alpha \dagger} K_{n m} \phi_{m}^{\beta} \\
K_{n m} & =K\left[\delta_{n, m+1} \gamma_{0}\left(i \gamma_{1}-r\right)-\delta_{n+1, m} \gamma_{0}\left(i \gamma_{1}+r\right)\right]+\delta_{n m}\left[M \gamma_{0}+i M_{5} \gamma_{1}\right]
\end{aligned}
$$

The interaction term becomes a little more complicated and has three parts

$$
H_{I}=\mathcal{E}_{I}+\frac{g^{2}}{2} \sum_{\alpha \beta} a_{\alpha}^{\dagger} \mathcal{N}^{\alpha \beta} a_{\beta}+\frac{g^{2}}{4} \sum_{\alpha \beta \gamma \delta} a_{\alpha}^{\dagger} a_{\beta}^{\dagger} \mathcal{M}^{\alpha \beta \gamma \delta} a_{\gamma} a_{\delta}
$$

where

$$
\mathcal{N}^{\alpha \beta}=\sum_{n m} \phi_{n}^{\alpha \dagger}|n-m| \phi_{m}^{\beta} \quad \mathcal{M}^{\alpha \beta \gamma \delta}=\sum_{n m} \phi_{n}^{\alpha \dagger} \phi_{n}^{\gamma}|n-m| \phi_{m}^{\beta \dagger} \phi_{m}^{\delta}
$$

and $\mathcal{E}_{I}$ is an unimportant constant $\mathcal{E}_{I}=-\frac{g^{2}}{4} \sum_{n m}|n-m|$. Note that loosely speaking, the constant term corresponds to the self-interaction of the Dirac sea-compensating charge, the quadratic term arises due to the interaction of this charge with the system and the quartic term represents the interactions of the system itself.

The mean energy in the Slater determinant (19) is a function of the set $S$ and is given by

$$
\mathcal{E}(S)=\mathcal{E}_{I}+\sum_{\alpha=1}^{L}\left[\mathcal{K}^{\alpha \alpha}+\frac{g^{2}}{2} \mathcal{N}^{\alpha \alpha}\right]+\frac{g^{2}}{4} \sum_{\alpha \beta=1}^{L}\left[\mathcal{M}^{\alpha \beta \beta \alpha}-\mathcal{M}^{\alpha \beta \alpha \beta}\right]
$$

The Hartree-Fock set of states $S^{H F}$ is now determined by minimizing $\mathcal{E}(S)$ with variables $\phi_{n}^{\alpha}$ subject to the orthonormality constraints. Standard manipulations then reveal that 
this variational problem can be solved by subjecting the one-particle wavefunctions to the $\mathrm{H}-\mathrm{F}$ equations of the form

$$
\sum_{m} \tilde{H}_{n m} \phi_{m}^{\alpha}=\epsilon^{\alpha} \phi_{n}^{\alpha}
$$

with

$$
\tilde{H}_{n m}=K_{n m}+\frac{g^{2}}{2}\left[V_{n m}^{D}+V_{n m}^{E}\right]
$$

and

$$
V_{n m}^{D}=\delta_{n m} \sum_{j}|n-j|\left(1-\sum_{\beta=1}^{L} \phi_{j}^{\beta \dagger} \phi_{j}^{\beta}\right) \quad V_{n m}^{E}=\sum_{\beta=1}^{L} \phi_{n}^{\beta}|n-m| \phi_{m}^{\beta \dagger}
$$

The "twidle" in $\tilde{H}_{n m}$ serves to denote the fact that these are not the one-particle matrix elements of Hamiltonian (9). As expected, the H-F equations take the form of a one-particle Hamiltonian eigenstate problem with the complication that the Hamiltonian matrix depends on the eigenstates themselves. Thus the equations have to be solved self-consistently. As usually, the self-consistent potential has direct and exchange parts.

Due to the self-consistent feature of the above H-F equations, finding an exact solution is a nontrivial task and I haven't succeeded in doing that. On the other hand, there is a simple way to attempt to solve these equations on finite lattices numerically, namely by iteration. Straightforward application of the iterative procedure however doesn't converge to the self-consistent solution. The nature of the problems is similar to those described in Ref. [19] in the context of continuum $Q C D_{2}$ in th large $N$ limit. I discuss these technical issues in Appendix. Using a modified approach, numerical solutions can be iteratively found in wide range of coupling constants.

Let me close this section with a few remarks concerning symmetry within the H-F approximation. It is usually helpful and desirable that the approximation scheme retains as much symmetry of the approximated system as possible. Especially if the main purpose of the investigation is spontaneous symmetry breaking. In particular, as discussed in the previous section, if $M_{5}=0$, the Hamiltonian (9) is invariant under parity. Is the parity invariance present in the approximation? In what sense?

The underlying dynamics driving the $\mathrm{H}-\mathrm{F}$ approximation is entirely embodied in the H-F equations. Therefore, the symmetries of these equations should also determine the 
symmetries of the approximation. It follows from transformation properties (14) that under the operation of parity the one-particle wave function $\chi_{n}$ transforms into $\gamma_{0} \chi_{L+1-n}$. Since the H-F Hamiltonian implicitly depends on its eigenstates, the parity operation in this case has to involve the whole set $S$. In fact, it can be checked quite easily that the operation

$$
S^{H F}=\left\{\phi_{n}^{\alpha}\right\} \longrightarrow P S^{H F}=\left\{\gamma_{0} \phi_{-n}^{\alpha}\right\}, \quad \epsilon^{\alpha} \longrightarrow \epsilon^{\alpha}
$$

is a symmetry of the H-F equations. In other words, if $S^{H F}$ is a self-consistent set solving (25), then $P S^{H F}$ is also a self-consistent set with corresponding one-particle energies equal.

Let me also mention that not just parity but all the discrete symmetries discussed in the previous section are preserved by the H-F approximation in the above sense. Of course, performing the symmetry operation on the H-F vacuum can lead to a Slater determinant involving different self-consistent set, thus opening the possibility of spontaneous symmetry breaking.

\section{Numerical Analysis (One Flavour)}

In this section, I will discuss the results of the H-F analysis for the model with one flavour. I will concentrate on the phase diagram in the $M-g^{2}$ plane. To observe the parity violating effects on a finite lattice, I fix $M_{5}$ to a very small value $\left(M_{5}=10^{-3}\right)$ throughout this section. The values of hopping parameter $(K=1)$ and Wilson parameter $(r=0.5)$ are set in such a way that the critical value of $M$ at zero coupling is $M_{c}(0)=1$. All quantities are given in the lattice units. Moreover, the electric field is allways measured in units of $g$. In particular, this expectation is calculated using formula (12) with operators of charge density replaced by their expectation values in the $\mathrm{H}-\mathrm{F}$ vacuum,

$$
\left\langle\rho_{n}\right\rangle^{H F}=\sum_{\alpha=1}^{L} \phi_{n}^{\alpha \dagger} \phi_{n}^{\alpha}-1
$$

and the factor of $g$ removed. 
To assess the accuracy of the self-consistent numerical solution and to provide the criterion for terminating the iterative procedure, I have computed

$$
\delta^{(k)}=\sum_{\alpha, n}\left|\phi_{n}^{\alpha(k+1)}-\phi_{n}^{\alpha(k)}\right|
$$

at each iterative step $k$. Here $\phi_{n}^{\alpha(k)} \in S^{(k)}$, the one-particle set after $k$ iterations (see Appendix). Obviously, $\delta=0$ only for the self-consistent set. In all cases discussed in this section $S^{(k)}$ has been accepted as a numerical solution only if $\delta^{(k)}<10^{-2}$. In most cases however, this number has been much smaller (up to four orders of magnitude at weak couplings). With the above bound, the physical characteristics of the H-F Slater determinant (such as energy), became essentially insensitive to further decrease of $\delta$. To achieve this accuracy on the lattices I have studied $(L=32,40,48)$ took typically a few tens of iterations at weak couplings $\left(g^{2} \leq 0.5\right)$ and a couple of hundreds at intermediate and strong couplings $\left(g^{2}>0.5\right)$. Working in the vicinity of the phase transition typically added roughly one order of magnitude to the number of iterations. In the region of couplings studied here $\left(g^{2} \leq 3\right)$, the self-consistent solution has always been straightforwardly found with free wave functions at given $M$ used as a starting point for the iteration.

The representative example of the most relevant finding in this study is displayed in Fig.1. The vacuum expectation value of the electric field in the middle of the 32 -site lattice is plotted as a function of the fermion mass at weak coupling $\left(g^{2}=0.1\right)$. Note that for large values of $M$, the electric field tends to zero as one would expect in the parity-invariant theory. However, at small fermion masses the field acquires an expectation value and the two regions are separated by a rapid transition. This suggests the existence of a parity violating phase transition and confirms the qualitative picture presented in Ref. [13] at the Hartree-Fock level.

The spatial dependence of the electric field across the lattice is plotted in Fig.2a for typical cases in the broken and symmetric phases. Note that in the broken phase, the field nicely settles to a uniform bulk value essentially across the whole lattice. In the symmetric example, the field is almost zero everywhere. It is quite interesting to see the spatial distribution of Hartree-Fock levels in these two situations. This is shown in Fig.2b where I plot the energy of these levels against the mean position of particles in them. In 
the symmetric case, all the particles reside on average in the middle of the lattice and the left-right symmetry is preserved up to small explicit violations caused by the presence of the small $M_{5}$-term. The filled levels produce a uniform charge distribution neutralized by the compensating charge. This is to be compared to the situation in the broken phase, where the left-right symmetry is completely lost. Indeed, it is energetically favourable for the levels to spread out asymmetrically. Filling the sea generates the surface charge and an electric field.

Similar behaviour is observed also at higher values of the gauge coupling. The resulting positions of phase transitions observed on the lattice with 48 sites are plotted in Fig.3. The transition points here are determined simply as the locations of the rapid rise of the vacuum expectation value of the electric field at fixed coupling. In particular, the phase transition is assumed to happen at the fermion mass $M_{c}\left(g^{2}\right)$, where this expectation value rises above $10^{-2}$, i.e. above the value one order of magnitude larger than the size of the parity violating $M_{5}$-term. By comparing to the results on smaller lattices $(L=32,40) \mathrm{I}$ expect the critical masses at nonzero couplings to be increased by a few parts per hundred in the infinite volume limit.

For the model in the standard Euclidean formulation, Gausterer and Lang [17] concluded the existence of a phase transition at infinite coupling. After appropriate rescaling the parameters of their model, the quoted position of this transition is $M_{c}(\infty) \simeq 0.32$. Although I don't know of any apriori reason why the phase transition should occur at the same place in both formulations, it is interesting to observe that their result is an acceptable asymptotic value at strong coupling here too.

Similarly to the electric field, the simplest local fermionic parity-odd operator, namely axial charge density $i \bar{\psi} \gamma_{5} \psi$, also acquires an expectation value at the parity violating phase transition. This is illustrated in Fig.4a where I plot both the electric field and the axial charge density as a function of fermion mass at $g^{2}=1.0$. Both operators appear to acquire an expectation value simultaneously as expected. Typical spatial dependence of the axial charge density in the broken and symmetric phases is plotted in Fig.4b, showing the bulk nature of the order parameter.

The relative size of the electric field and the axial charge density in the broken phase 
varies with gauge coupling. This is demonstrated in Fig.5 where I plot these expectation values at fixed fermion mass. Note that while the electric field starts up finite at weak coupling and decreases monotonically at intermediate and strong couplings, axial charge density behaves in a complementary way. It approaches zero with vanishing coupling and rises as the coupling increases.

The above behaviour of electric field is simply a manifestation of charge shielding, an effect well known to be present in the continuum theory as well. Indeed, consider first the free theory. In that case it is just the filled surface mode that is responsible for the parity-breaking effects. That's why the electric field approaches value $0.5(\theta=\pi)$ when coupling tends to zero. However, once the gauge coupling is turned on, the rest of the levels spread out (see Fig.2b) and the accumulation of surface charge is a result of the collective action of all self-consistently interacting particles in filled states. The net effect of this phenomenon is a screening of the surface charge. As the value of the gauge coupling increases, while remaining in the broken phase, one expects the levels to spread out and screen even more since the system wants to reduce the positive attraction energy of the surface charges. For example, at $g^{2}=1.5$, the spatial distribution of $\mathrm{H}-\mathrm{F}$ levels is shown in Fig.6. At strictly strong coupling, when the interaction term absolutely dominates, every particle in the sea will live bound to just one site of the lattice, thus eliminating the surface charge completely. Therefore, the field is expected to vanish in this limit in the $\mathrm{H}-\mathrm{F}$ approximation.

In the light of the above considerations, behaviour of the axial charge density in Fig.5 becomes also quite natural. Indeed, since at zero coupling the parity violation is all concentrated on the ends, it will not be reflected in the expectation value of the local operator inside the system. Consequently, one expects the bulk axial density to vanish. At strong coupling however, parity violation is equally contributed by all the filled levels and the axial density acquires an expectation value.

An interesting feature already present in the examples of Fig.2, but quite striking in Fig.6, is that because of the interaction energy, it is not necessarily the lowest one-particle states that are filled to form the H-F vacuum. Indeed, in Fig.6 almost half of the filled levels (denoted by diamonds) are those with positive one-particle energies. This is discussed in 
more detail in the Appendix.

Finally, let me close this section by discussing the order of the parity violating phase transition in the H-F approximation. It is well known that the mean field-like approaches are frequently misleading about the order of the phase transition and the critical exponents. Therefore, while I assume that it is plausible for H-F approximation to recognize the transition, the information it gives about the order should be taken with some care.

I have calculated the connected correlation functions for electric field and axial charge density in the H-F vacua. For the case of electric field, these functions have a very nice exponential decay in all cases I have studied and the corresponding correlation lengths could be reliably determined. Typical behaviour of the inverse correlation length (mass gap) across the phase transition is shown in Fig.6a. In the symmetric phase, the correlators of the axial charge behave in the same way. However, in the broken phase they show some differences as can be observed from Fig.6a. For weak couplings, the determination of the correlation length from these axial charge correlators in the broken phase was less accurate than from the electric ones. Using the electric field correlation functions, Fig.6b shows the mass gap along the line of phase transitions on a lattice of 48 sites. These results are reasonably finite-size stable (more so at stronger couplings) and I exclude the possibility of mass gap reducing to zero in the infinite volume limit. I conclude that in the H-F approximation the phase transition is of first order at finite $g$, approaching a second order endpoint at zero coupling.

\section{Two Flavours}

Turning now to the case of two degenerate fermion flavours, I will consider the lattice Hamiltonian

$$
H=\sum_{f} \sum_{n m} \psi_{n}^{f \dagger} K_{n m} \psi_{m}^{f}-\frac{g^{2}}{4} \sum_{f h} \sum_{n, m} \rho_{n}^{f}|n-m| \rho_{m}^{h} .
$$

Here $f, h$ are flavour indices assuming two values, $K_{n m}$ is defined in (21) and

$$
\rho_{n}^{f}=\psi_{n}^{f \dagger} \psi_{n}^{f}-1
$$


The fermionic operators $\psi_{n}^{f}$ are subject to the canonical anticommutation relations.

Note that with flavours being degenerate, the parameter space of this two-flavour theory is the same as for a single flavour, namely $M, M_{5}, g$. Also, similarly to the one-flavour case, the above lattice model retains all the discrete symmetries of the corresponding continuum theory. In addition, the two-flavour model is invariant under unitary transformations in flavour space. All of these symmetries will be preserved by the Hartree-Fock approximation in the sense discussed in Sec. III.

Inclusion of the flavour index does not require any conceptual changes in the application of the Hartree-Fock procedure. On the technical side, it is easiest to skip the explicit use of flavour notation and assemble the two fermionic operators on site $n$ into a 4-component column $\psi_{n} \equiv\left(\begin{array}{c}\psi_{n}^{1} \\ \psi_{n}^{2}\end{array}\right)$. The Hamiltonian then takes the form

$$
H=\sum_{n m} \psi_{n}^{\dagger} \tilde{K}_{n m} \psi_{m}-\frac{g^{2}}{4} \sum_{n, m} \rho_{n}|n-m| \rho_{m}
$$

with

$$
\tilde{K}_{n m}=\left(\begin{array}{cc}
K_{n m} & 0 \\
0 & K_{n m}
\end{array}\right)
$$

and

$$
\rho_{n}=\psi_{n}^{\dagger} \psi_{n}-2
$$

Note that to compensate for the charge of the Dirac sea now requires two units of charge per site.

Using the above notation and the decomposition

$$
\psi_{n}=\sum_{\alpha=1}^{4 L} a_{\alpha} \phi_{n}^{\alpha}
$$

the derivation of the $\mathrm{H}-\mathrm{F}$ equations is a line by line repetition of the procedure for the one flavour case up to the factors of two coming from the doubling of the compensating charge. Indeed, the H-F equations take the form

$$
\sum_{m} \tilde{H}_{n m} \phi_{m}^{\alpha}=\epsilon^{\alpha} \phi_{n}^{\alpha}
$$

with

$$
\tilde{H}_{n m}=K_{n m}+\frac{g^{2}}{2}\left[V_{n m}^{D}+V_{n m}^{E}\right]
$$


and

$$
V_{n m}^{D}=\delta_{n m} \sum_{j}|n-j|\left(2-\sum_{\beta=1}^{2 L} \phi_{j}^{\beta \dagger} \phi_{j}^{\beta}\right) \quad V_{n m}^{E}=\sum_{\beta=1}^{2 L} \phi_{n}^{\beta}|n-m| \phi_{m}^{\beta \dagger} .
$$

Note that these equations are formally almost identical to those for one flavour. The crucial difference however is that $\phi_{n}^{\alpha}$ is now a 4 -component object and $\tilde{H}$ a $4 L \times 4 L$ complex matrix.

\section{Numerical Analysis (Two Flavours)}

I will start with the discussion of the phase structure in the $M-M_{5}$ plane at fixed gauge coupling. Before turning to the results of the $\mathrm{H}-\mathrm{F}$ analysis, let me first briefly explain what one would expect to be happening here at weak coupling based on the surface mode picture. To do that, I will need to borrow the of ideas of Ref. [2], and to make this paper reasonably self-contained, to review briefly the part that is relevant here. Thinking first in the continuum context, consider the standard mass term $m \bar{\psi} \psi$ and its transformation under the chiral rotation $\psi \rightarrow e^{i \theta / 2 \gamma_{5}} \psi$. We have

$$
m \bar{\psi} \psi \longrightarrow m \cos (\theta) \bar{\psi} \psi+m \sin (\theta) i \bar{\psi} \gamma_{5} \psi
$$

Therefore, the chiral rotation by angle $\theta$ corresponds to the rotation of the vector $(m, 0)$ in the $m-m_{5}$ plane around the origin ("chiral point") by the same angle $\theta$. While naively expecting that the physics should be the same after the above change of variables, this is actually not the case because of the chiral anomaly. What we are actually getting is a

physics with different "gauge" $\theta$-parameters, i.e. with different background electric field, realized for example through the existence of the surface charges. With this identification, the above transformation prescription gives the approximate relation (the renormalization effects, for example, will shift the chiral point to negative $m$ ) between the theory considered in the parameter spaces $(e, m, \theta)$ and $\left(e, m, m_{5}\right)$.

On a lattice with Wilson fermions, the situation is a little more complicated, because except from $\left(M_{c}, 0\right)$, there is another chiral point in the $M-M_{5}$ plane, namely $\left(-M_{c}, 0\right)$, where the doubler goes massless. The conjecture then is that here the total $\theta$-parameter 
gets two contributions, each being the angle with respect to the two chiral points, with doubler's contribution taken with the reversed sign. These angles are sketched in Fig.8a. $N_{f}$ degenerate flavours will contribute equally to the total value of $\theta$ and consequently, one expects the following approximate relation to hold at weak coupling

$$
\theta=N_{f}\left(\theta_{p}-\theta_{d}\right)
$$

Here $\theta_{p}, \theta_{d}$ are the contributions of the particle and the doubler respectively. In other words, on a lattice with open boundaries, the system is expected to generate surface charges in such a way, that the resulting electric field will approximately correspond to $\theta$ given by the above relation.

If this qualitative picture is correct, there should be phase transitions occuring in the $M-M_{5}$ plane at the positions where $\theta$ reaches $\pi$. Indeed, for $\theta>\pi$, it will be energetically favourable to create a fermion-antifermion pair thus reducing the magnitude of the electric field and switching its sign. Consequently, $\theta$ should jump from $\pi$ to $-\pi$ across these phase transitions. The condition $\theta=\pi$ defines a line in $M-M_{5}$ plane, but its qualitative behaviour strongly depends on the number of flavours. With single flavour, the only solution is a straight line, connecting $M_{c}$ and $-M_{c}$. This can be understood already from the point of view of the "naive" surface mode picture as I presented in the Introduction. Indeed, switching the sign of a small parity violating " $M_{5}$ "-term causes the two surface modes to exchange the ends of the lattice, thus switching the sign of the surface charges and the electric field. For two flavours, the condition $\theta=\pi$ defines a circle with centre at the origin and radius $M_{c}$. Including a small flavour breaking in both $M$ and $M_{5}$ to visualise the chiral endpoints, the phase diagram is expected to look qualitatively as sketched in Fig.8b [2]. Note that contrary to the single flavour case, the prediction of this phase structure in the $M-M_{5}$ plane is quite nontrivial.

Guided by this simple picture at weak coupling, I set out to look for these phase transitions in the Hartree-Fock approximation. For numerical work, I again fixed the values of the hopping parameter $(K=1)$ and the Wilson parameter $(r=0.5)$, so that the critical value of $M$ at zero coupling is $M_{c}(0)=1$. Also, I have always included a very small explicit flavour breaking in both $M$ and $M_{5}$. In particular, the masses of flavours were 
of the form $M \pm 0.001$ and $M_{5} \pm 0.001$. The self-consistent solutions were again obtained by the modified iteration procedure as described in Appendix. For all results presented here the accuracy of the solution, given by Eq. (30), was better than $10^{-3}$ and typically about $10^{-6}$. To achieve this accuracy in the immediate vicinity of the phase transition on a lattice of 40 sites took less then $10^{4}$ iterations at strongest coupling studied here $\left(g^{2}=6\right)$.

The graphs in Figs.9a,b illustrate how the above qualitative ideas are reflected in the Hartree-Fock approximation. In Fig.9a I plot the vacuum expectation value of the electric field along the $M_{5}$-axis $(M=0)$ at $g^{2}=0.1$ on a lattice with 40 sites. Note that the H-F vacuum nicely exhibits the expected abrupt change in the electric field and the reversal of its sign. Probing the field along the $M$-axis (with small $M_{5}$ present) gives the dependence ploted in Fig.9b. While behaving in qualitatively the same way, the magnitude of the field is becoming small as one approaches the $M-g^{2}$ plane. This is what one would expect if these $\theta=\pi$ transitions, which are naturally first order, end in a second order chiral endpoint in this plane. The anlysis of the electric field correlators suggests however, that in the H-F approximation, these transitions at finite coupling are first order even close to the $M-g^{2}$ plane. It should be stressed again however, that this might well be an artifact of the approximation.

Defining the transition point as the position in the $M-M_{5}$ plane where the field switches its sign, I plot the phase diagrams for $g^{2}=0.1$ and $g^{2}=1.2$ on a lattice with 32 sites in Fig.10. In fact, only the points in the upper right quadrant were really calculated. The rest of them were obtained using symmetry with respect to the mass reflections. Note that while not exactly of circular shape, the transition lines reflect the expected qualitative features deduced from the surface mode picture. Also, at $g^{2}=0.1$, non-negligible finitesize effects are present here. While the transition point along the $M$-axis is essentially stable against the increase of the lattice size, the transitions along the $M_{5}$-axis occur at $M_{5}=0.36,0.42,0.48$ on the lattices with 32,40 and 48 sites respectively. Thus it is quite possible that the ellipse-like shape of the transition line will become more circle-like in the infinite volume limit.

Let me now turn to the question of Aoki's phase in the $M-g^{2}$ plane. First note that the surface mode picture doesn't suggest that parity-flavour broken phase should exist here 
at weak coupling. Indeed, as one turns off the $M_{5}$ and moves along the $M$-axis, the angle $\theta$ defined by (41) is always zero. Both flavours will generate their surface mode as $M$ is lowered below $M_{c}$, thus changing $\theta$ by $2 \pi$ and physically changing nothing. A good way to picture this is by looking at Fig.8b: However small the flavour breaking is, if one moves close enough to the $M$-axis, it is always possible to pass below the chiral point.

Discussion of these issues in two dimensions might appear a little academic since because of the Mermin-Wagner theorem [20], one would not expect flavour to be spontaneously broken here at any coupling. Nevertheless, if the surface mode picture is of relevance to QCD in four dimensions, then this qualitative prediction wouldn't change. Moreover, I consider the following to be good reasons to investigate these issues in the context of the Schwinger model itself: 1) The lattice model, defined by (33), is nonlocal, and as such does not exactly satisfy the usual assumptions of the theorem. 2) Parity can still be broken. 3) Flavour could be erroneously broken within the H-F approximation. Then, although not useful as an information about the lattice Schwinger model, it can serve as a toy picture of what might be happening in QCD where there is quite convincing evidence that parity-flavour is broken at strong enough coupling.

With that in mind, I have calculated the expectation values of $\bar{\psi} \gamma_{5} \tau_{3} \psi$ and $\bar{\psi} \gamma_{5} 1 \psi$ in the $\mathrm{H}-\mathrm{F}$ vacua. Here $\tau_{3}$ is the third Pauli matrix and 1 a unit matrix in flavour space. Note that the form of flavour breaking in $M_{5}$ used here chooses the $\tau_{3}$-direction if flavour is broken. Note also that if $M_{5}=0$ and $\left\langle\bar{\psi} \gamma_{5} \tau_{3} \psi\right\rangle \neq 0$, it is both parity and flavour that are spontaneously broken. On the other hand, if this expectation is nonzero at nonzero $M_{5}$, the parity is broken explicitly while the flavour spontaneously. Furthermore, if at $M_{5}=0$ we had $\left\langle\bar{\psi} \gamma_{5} 1 \psi\right\rangle \neq 0$ and $\left\langle\bar{\psi} \gamma_{5} \tau_{3} \psi\right\rangle=0$, it would indicate that only parity has been spontaneously broken.

The results of the H-F analysis in $M-g^{2}$ plane (with $M_{5}=10^{-2}$ ) on the lattices with up to 40 sites are as follows. I have found no evidence of $\bar{\psi} \gamma_{5} 1 \psi$ acquiring an expectation value in the region of couplings $g^{2} \leq 6$. Consequently, there is no indication of parity being broken alone. However, there are regions where $\left\langle\bar{\psi} \gamma_{5} \tau_{3} \psi\right\rangle$ is nonzero in the Hartree-Fock approximation on the finite lattice. For example, in Fig.11a I plot this expectation as a function of fermion mass at $g^{2}=1.2$. The broken region appears as a narrow peak adjacent 
from the left to the " $\theta=\pi$ " $*$ transition point on this lattice of 40 sites. I have observed similar peaks at $g^{2}=0.1$ and $g^{2}=4.0$ with heights roughly 0.08 and 0.70 respectively. For the two weaker of the above couplings, I have also performed a finite-size analysis of the width of this broken region. This width decreases linearly with $1 / L$, exhibiting a small negative intercept in both cases. On the other hand, the heights of the peaks stay constant as the lattice size increases. I therefore conclude that these narrow regions will not survive in the H-F phase diagram in the infinite volume limit. The only remnant of them will probably be the singular behaviour of the parity-flavour order parameter at the " $\theta=\pi$ " transition point.

The situation qualitatively changes at even stronger couplings. In particular, the parity-flavour broken phase indeed opens up at the subcritical fermion masses. This is demonstrated in Fig.11b, where I show the behaviour of the order parameter at $g^{2}=6.0$ on a lattice with 32 sites. There has been a negligible change here as the lattice size increased to $L=40$. I therefore expect the finite-size effects to be small. It is also worth mentioning that $\left\langle\bar{\psi} \gamma_{5} \tau_{3} \psi\right\rangle \neq 0$ in the whole inside region of the " $\theta=\pi$ " line at this strong coupling and not only in the $M-g^{2}$ plane. This is in contrast to the case of narrow broken regions at weaker couplings which can only be observed close to $M-g^{2}$ plane on a finite lattice.

This concludes the review of the most important aspects of the numerical information obtained in this study. In the last section, I will turn to generalizations and speculations.

\section{Summary, Generalizations and Speculations}

The Schwinger model on a lattice with Wilson fermions has been studied in the Hartree-Fock approximation. The main focus was given to the global structure of phase diagrams with one and two degenerate flavours of fermions. In future communication, I plan

* Note that I loosely refer to a transition, where electric field switches the sign as the " $\theta=\pi "$ transition even at strong coupling. This should not be taken too literally neither here, nor in what follows. 
to report on the study of the continuum limit in this framework. The surface mode picture $[2,13]$ served as a reliable guide in these investigations at weak coupling. The nonperturbative nature of H-F approximation however, allows to study the model at intermediate and strong couplings as well.

For the case of a single flavour, I plot in Fig.12 the qualitative behaviour of the concluded full phase diagram of the model in the Hartree-Fock approximation. There is a planar region, embedded in the $M-g^{2}$ plane, where parity is spontaneously broken. From the point of view of the surface mode picture, it can be understood as the surface of " $\theta=\pi$ " transitions. Entering the region from the $M$-direction is accompanied by the appearance of the surface charges and the background electric field (" $\theta=0 \rightarrow \theta= \pm \pi$ "). Crossing the region in the $M_{5}$-direction corresponds to reversing the sign of the electric field (" $\theta=\pi \leftrightarrow \theta=-\pi$ "). In accordance with Aoki's scenario, $\bar{\psi} \gamma_{5} \psi$ acquires an expectation value in the broken region. Taking into account the infinite-coupling result of [17], I expect the parity-violating phase to extend all the way to $g^{2} \rightarrow \infty$.

While the " $\theta=\pi \leftrightarrow \theta=-\pi$ " transitions are naturally first order, there is a strong evidence that the parity-violating phase transitions in $M-g^{2}$ plane are also first order in the Hartree-Fock approximation at nonzero $g$. This seems quite unnatural since one would have the first order ends at the boundaries of the parity-violating region. On the other hand, if one thinks conventionally about taking the chiral continuum limit, the first order transition looks quite appropriate. There are two parts to the conventional wisdom about taking this limit, which is usually thought about in analogy to QCD in four dimensions. First, since the gauge coupling constant is dimensionful (inverse length) in two dimensions, it is assumed that the continuum limit can only be taken at vanishing $g$ (the dimensionless lattice coupling). Second, the chiral limit is assumed to be taken by following the line of phase transitions $M_{c}\left(g^{2}\right)$ towards $g=0$. The immediate consequence of these assumptions is that $g(a)$ vanishes at $a=0$ and is an increasing function in the vicinity of this point. Here $a$ is the lattice spacing. Denoting by $\Delta(a)$ the dimensionless mass gap along the line of phase transitions, the physical mass of the lightest particle in the theory is given by $\Delta(a) / a$. Since chiral symmetry is broken in the continuum and the lowest mass is nonzero $(e / \sqrt{\pi})$, the above ratio should approach the constant positive value as $a \rightarrow 0$. 
Consequently, similarly to $g(a), \Delta(a)$ should also vanish at $a=0$ and increase in the vicinity of this point. Puting the above two conclusions together, $\Delta(g)$ must have this local property as well. In particular, it is zero at $g=0$, but increases as $g$ becomes finite. Hence, if the conventional picture about chiral continuum limit is correct, the transitions should become first order as the gauge coupling is turned on.

In the light of the above considerations, it is not entirely obvious that the H-F approximation is giving an incorrect answer here (which it of course well can). One possible solution is that what is depicted in Fig.12 is not all that happens in the model. In particular, there could be another sheet of first order phase transitions going off the $M-g^{2}$ plane and crossing this plane at $M_{c}\left(g^{2}\right)$. If that was the case, then the second order ends at $M_{c}\left(g^{2}\right)$ would not appear to be necessary. However, I have not found the evidence that would support this scenario in the H-F approximation. In summary, the order of parity violating phase transition is a very interesting issue by itself. However, it can only be satisfactorily settled by accurate calculation beyond the H-F approximation.

Similarly to the one-flavour case, there is a surface of " $\theta=\pi$ " transitions also in the model with two flavours. It takes a more complicated shape however and its qualitative behaviour in the H-F approximation is depicted in Fig.13a. The "tube" of phase transitions encloses the $g^{2}$-axis as the explicit flavour breaking is taken to zero and touches the $M-g^{2}$ plane at $M_{c}\left(g^{2}\right)$. Since the electric field generated by the surface charges switches the sign, the transitions across the surface of the "tube" are naturally first order. In the H-F approximation, this is so even when approaching $M_{c}\left(g^{2}\right)$ at finite coupling. The transition close to $M_{c}\left(g^{2}\right)$ becomes second order in H-F approximation only as $g \rightarrow 0$. Note also that contrary to the single-flavour case, the possible continuous nature of the phase transitions along $M_{c}\left(g^{2}\right)$ would not be in conflict with the above argument concerning the chiral continuum limit. This is because in the multi-flavour case, the nonsinglet part of the flavoured chiral symmetry is not anomalous and there is a massless particle in the continuum theory.

The crucial difference between Fig.12 and Fig.13a is that with two flavours, it is only $M_{c}\left(g^{2}\right)$ that is shared by the $M-g^{2}$ plane and the surface of " $\theta=\pi$ " transitions. If the identification of the " $M_{5}$ "-physics on the lattice and the " $\theta$ "-physics in the continuum is 
correct at weak coupling, nothing special should happen upon crossing $M_{c}\left(g^{2}\right)$ with respect to parity, and it indeed doesn't. At strong coupling however, the above scenario might well break. This is nicely observed in the H-F approximation. In particular, the numerical evidence suggests the existence of rather strong coupling $g_{s}\left(4<g_{s}^{2}<6\right)$, so that for $g>g_{s}$, the expectation value of $\bar{\psi} \gamma_{5} \tau_{3} \psi$ is nonzero inside the "tube" of Fig.13a. Consequently, parity-flavour is broken in $M-g^{2}$ plane at subcritical masses and strong couplings. I conclude the qualitative H-F phase diagram in this plane as depicted in Fig.13b. The full lines in this phase diagram represent $M_{c}\left(g^{2}\right)$ and they are also characterized by the fact that $\langle\bar{\psi} \psi\rangle$ exhibits a jump as they are crossed. Parity-flavour however, is only broken in the "BP"-region, bounded from bellow by the dashed line.

The above results indicate that Aoki's scenario is not realized in the two-flavour Schwinger model at the H-F level. It is quite feasible, that this is the case for QCD in four dimensions as well $[21,22]$. Although the analogy between $Q E D_{2}$ and $Q C D_{4}$ should certainly not be taken too seriously (especially in case of an approximation), I believe that the phase diagram of Fig.13b indeed represents a possible toy picture of what might be happening in the latter case. In particular, that the parity-flavour broken phase shrinks to zero width before entering the vicinity of the continuum limit. There would still be a line $\kappa_{c}\left(g^{2}\right)$, running up from the QCD fixed point, on which a transition in $\langle\bar{\psi} \psi\rangle$ could be observed. However, $\left\langle\bar{\psi} \gamma_{5} \tau_{3} \psi\right\rangle$ would remain zero.

\section{Appendix}

In this appendix I will briefly describe a technical detail on the numerical procedure used to solve the H-F equations (37). The standard way to proceed is to iteratively generate the sequence of sets of one particle states $\left\{S^{(0)}, S^{(1)}, \ldots S^{(k)}, \ldots\right\}$, so that $S^{(k+1)}$ is the eigenset of $\tilde{H}^{(k)}$. Here $\tilde{H}^{(k)}$ is the H-F Hamiltonian with direct and exchange potentials

determined from wavefunctions of $S^{(k)}$. With a reasonable choice of the initial set the sequence frequently converges well to the self-consistent set $S_{H F}$.

Note however, that there is certain ambiguity in the procedure that might cause a problem. It arises because of the fact that the energy of the H-F vacuum is not just a 
sum of the one particle energies of the filled levels. Indeed, at nonzero coupling there is an interaction part contributing to the total energy and it may well be that the filled levels are not those from $S^{H F}$ with lowest one-particle energies. If that is the case and the iteration proceeds by filling the lowest levels at each step, the procedure can never converge to a self-consistent set.

This bad looking flaw can however be quite easily rectified [19]. Instead of the original H-F problem $(24,37)$, consider the one with the two body potential shifted by a constant, i.e.

$$
|n-m| \longrightarrow|n-m|+C \text {. }
$$

One naturally expects that a resulting H-F vacuum will not be physically different from that of the original problem. Indeed, it can be easily checked that both problems share their solutions. However the vacuum energy and also the one particle energies will change. In particular,

$$
\begin{aligned}
E & \longrightarrow E+C L \frac{g^{2}}{4} \\
\epsilon^{\alpha} & \longrightarrow \begin{cases}\epsilon^{\alpha}+C \frac{g^{2}}{2}, & \alpha \text { filled; } \\
\epsilon^{\alpha}, & \alpha \text { empty. }\end{cases}
\end{aligned}
$$

Note that it is only the filled levels that get shifted in energy, not the empty ones. Therefore by choosing $C$ to be negative and sufficiently large one can allways make the filled levels to be those with lowest one-particle energies. The H-F problem with such $C$ can then in principle be solved by standard iteration as described above. If the solution is found, it is also the $\mathrm{H}-\mathrm{F}$ vacuum of the original problem.

In an actual computation, the constant $C$ was chosen by trial and error. If the iteration failed for a given $C$, a larger value has been set. In general, larger values were needed for larger values of $g$, as one would expect. 


\section{Acknowledgements}

I am indebted to Mike Creutz for many valuable discussions and for helping me to rectify some of the lingual shortcomings in the preliminary version of this paper. I have benefited from discussions with Tony Kennedy, Urs Heller, Khalil Bitar, Claudio Rebbi and Jorge Piekarewicz. Thanks also to John Negele for bringing the Ref. [19] into my attention. The financial support from DOE under Grant Nos. DE-FG05-85ER250000 and DEFG05-92ER40742 is also acknowledged.

\section{References}

1. H. Nielsen, M. Ninomiya, Nucl. Phys. B185 (1981) 20; Nucl. Phys. B193 (1981) 173.

2. M. Creutz, Nucl. Phys. B (Proc. Supl.) 42, 56 (1995).

3. Y. Shamir, TUAP-2287-95, hep-lat/9509023.

4. S. Treiman, R. Jackiw, B. Zumino, and E. Witten, Current algebra and anomalies, (World Scientific, 1985, QC793.3.A4C87).

5. L. Karsten, J. Smit, Nucl. Phys. B183 (1981) 103; A. Coste, C. Korthals-Althes, O. Napoly, Phys. Lett. B179 (1986) 125.

6. S. Aoki, Phys. Rev. Lett. 57 (1986) 3136; Nucl. Phys. B314 (1989) 79.

7. S. Aoki, A. Gocksch, Phys. Rev. D45 (1992) 3845.

8. S. Aoki, A. Ukawa and T. Umemura, UTHEP-313, Aug 1995; hep-lat/9508008.

9. S. Aoki, S. Boettcher, A. Gocksch, Phys. Lett. B331 (1994) 157.

10. K. Bitar, P. Vranas Phys. Rev. D 50 (1994) 3406.

11. D. Kaplan, Phys. Lett. B288 (1992) 342.

12. K. Jansen, DESY-94-188, hep-lat/9410018.

13. M. Creutz, I. Horváth Phys. Rev. D 50 (1994) 2297.

14. S. Coleman, Ann. Phys. 101 (1976) 239.

15. C. R. Gattringer, E. Seiler, Ann. Phys. 233 (1994) 97; J. E. Hetrick, Y. Hosotani, S. Iso, Phys. Lett. B350 (1995) 92. 
16. E. Marinari, G. Parisi, C. Rebbi, Nucl. Phys. B190 [FS3] (1981) 734; S. R. Carson, R. D. Kenway, Ann. Phys. 166 (1986) 364.

17. H. Gausterer, C. H. Lang, Phys. Lett. B341 (1994) 46.

18. V. Azcoiti et al., INFN-LNF 94/009(P); hep-lat/9401032.

19. L. L. Salcedo, S. Levitt, J. W. Negele, Nucl. Phys. B361 (1991) 585.

20. N. D. Mermin, H. Wagner Phys. Rev. Lett. 17 (1966) 1133.

21. M. Creutz, BNL-62123, May 1995; hep-th/9505112.

22. K. Bitar, private communication. 


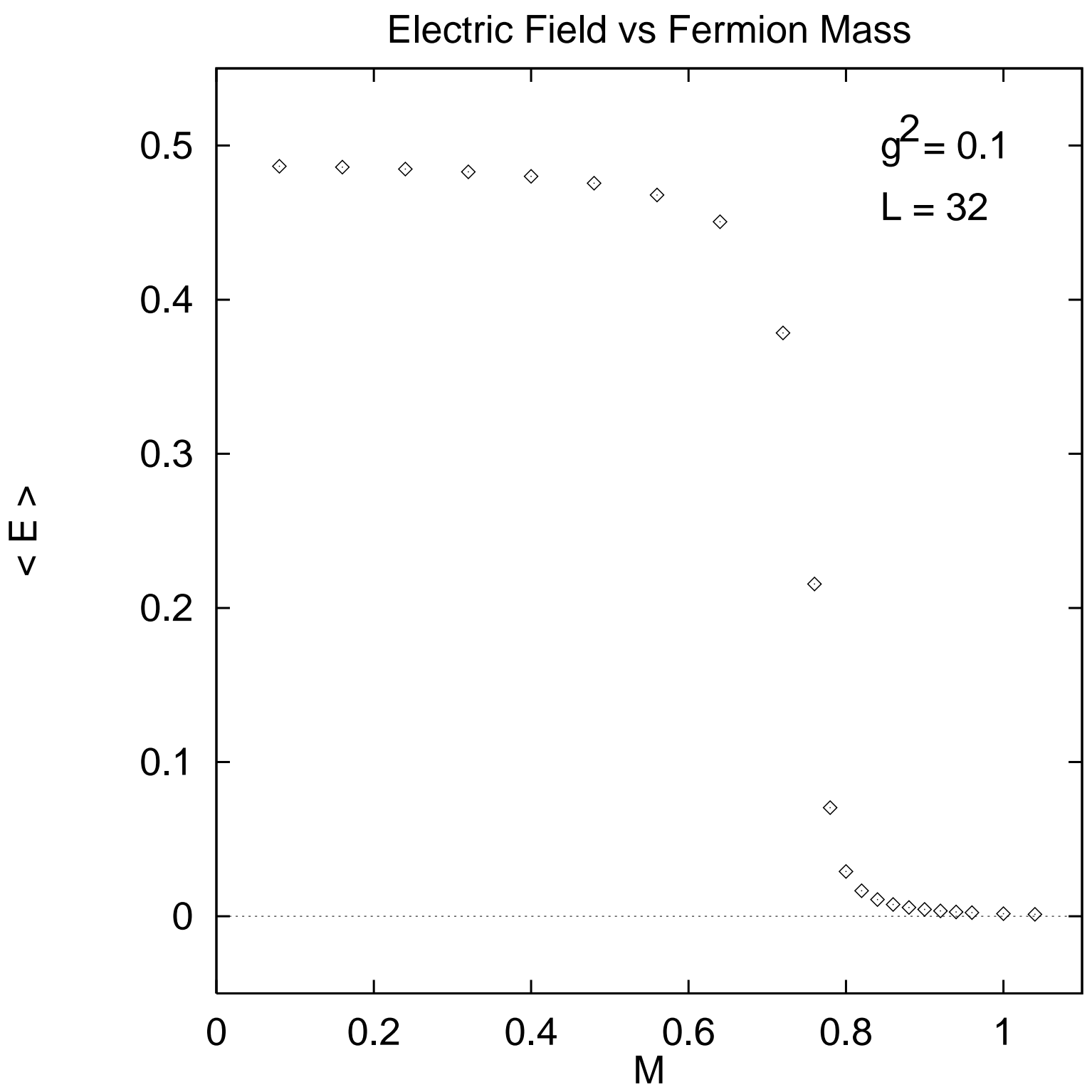

Fig.1 Vacuum expectation value of the electric field in the middle of the system as a function of fermion mass. 

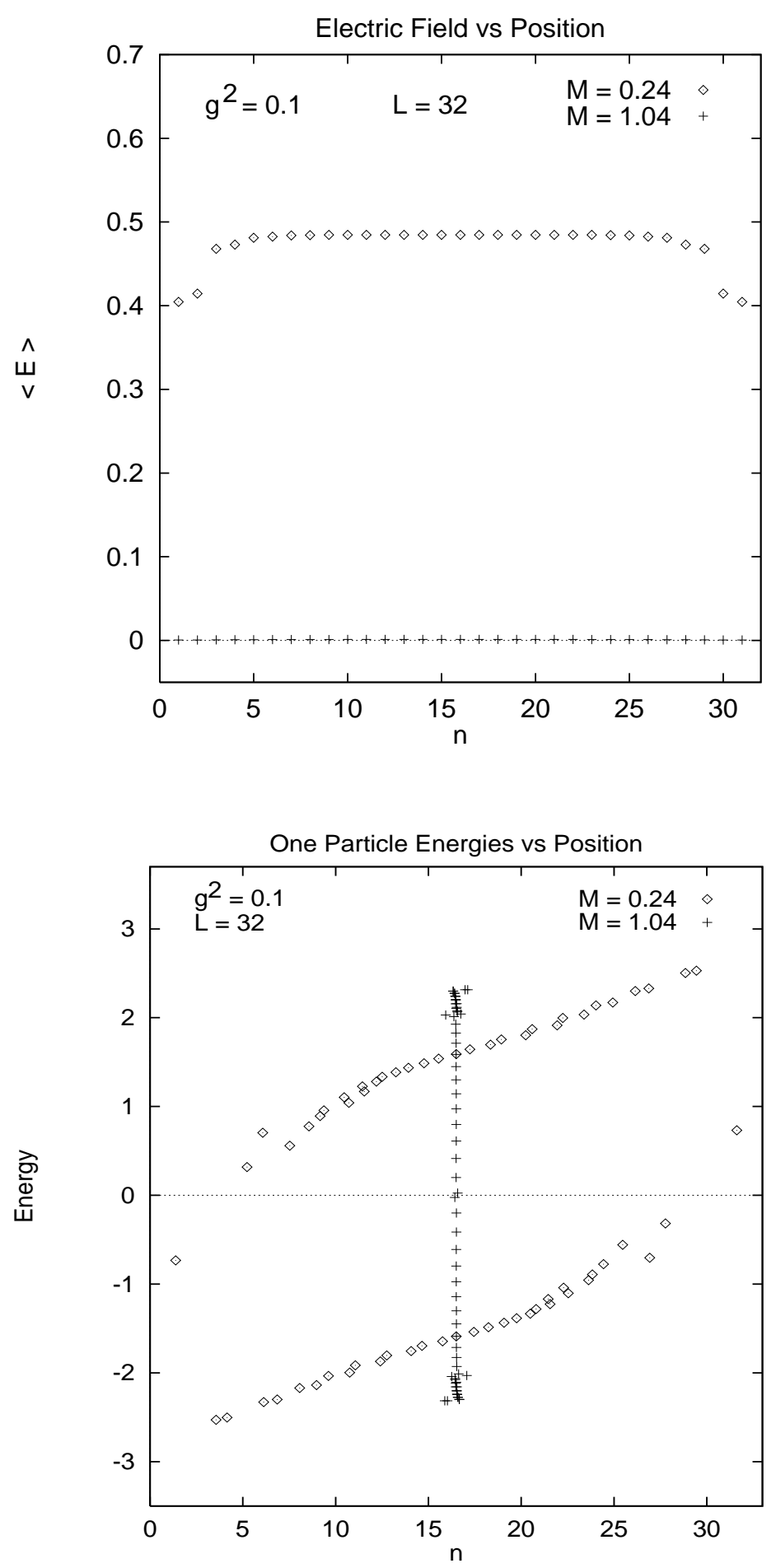

Fig.2a (Upper) Spatial dependence of the electric field in symmetric $(M=1.04)$ and broken $(M=0.24)$ phases. Fig.2b (Lower) Spatial distribution of the corresponding $\mathrm{H}-\mathrm{F}$ levels. 


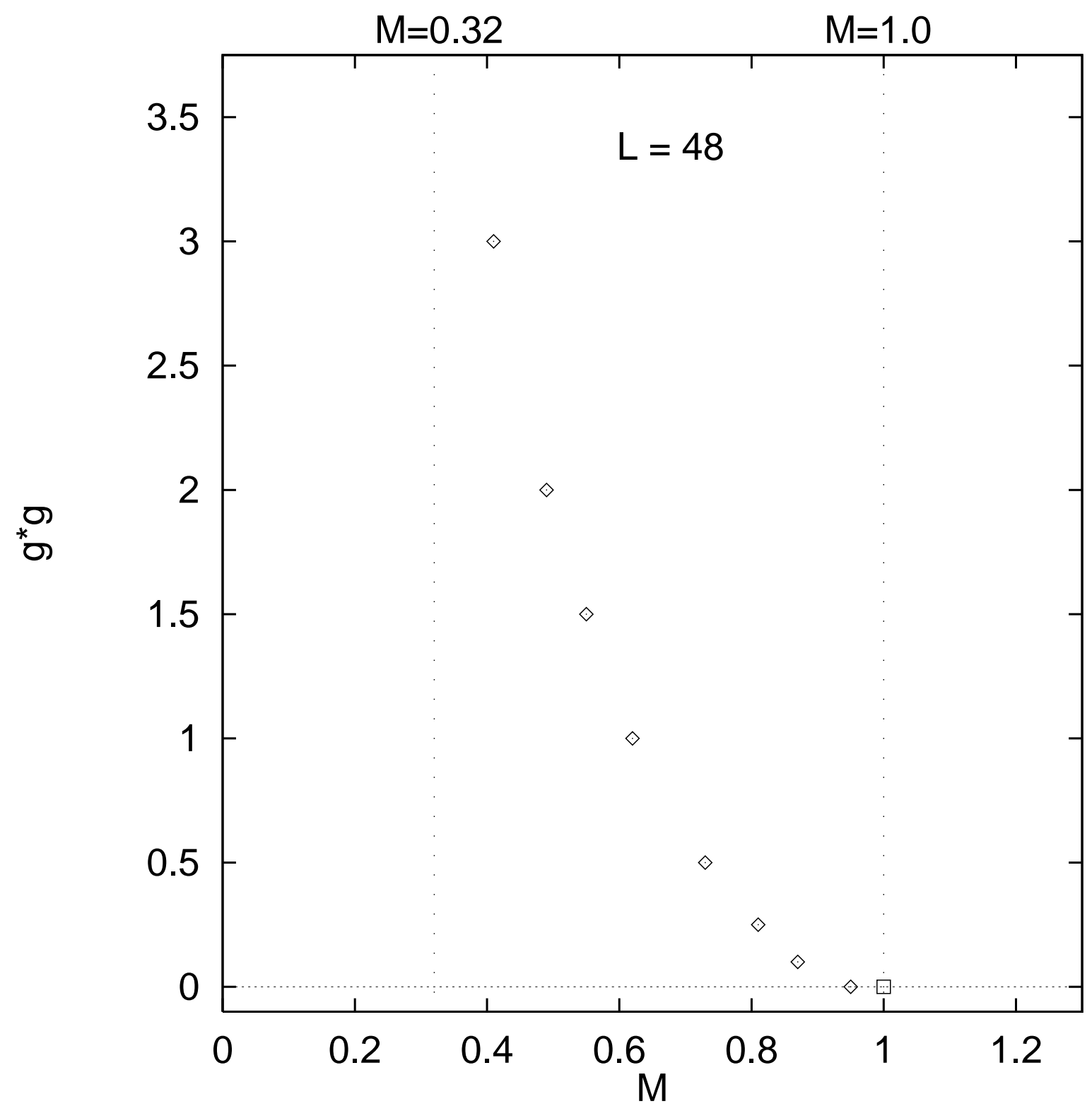

Fig.3 Hartree-Fock phase diagram in $M-g^{2}$ plane as seen on a finite lattice. Diamonds sample the critical line $M_{c}\left(g^{2}\right)$, with parity broken in the left region. The square marks $M_{c}(0)$ at infinite volume limit. The left vertical line represents $M_{c}(\infty)$ quoted in [17] for the model in standard Lagrangian formulation. 

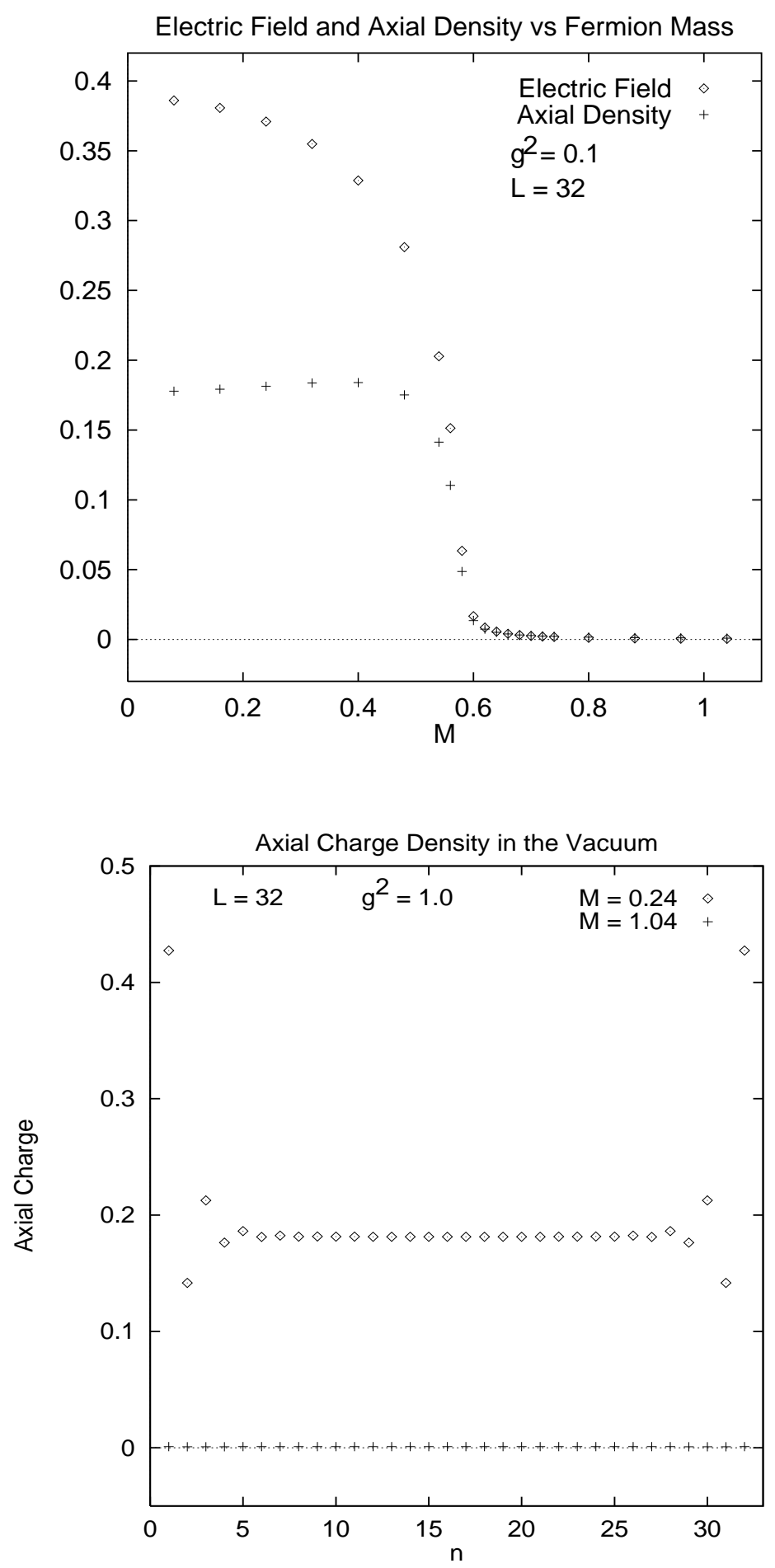

Fig.4a (Upper) Electric field and axial charge density against the fermion mass. Transitions seem to occur simultaneously. Fig.4b (Lower) Spatial dependence of axial density in symmetric $(M=1.04)$ and broken $(M=0.24)$ phases. 


\section{Electric Field and Axial Density in Broken Phase}

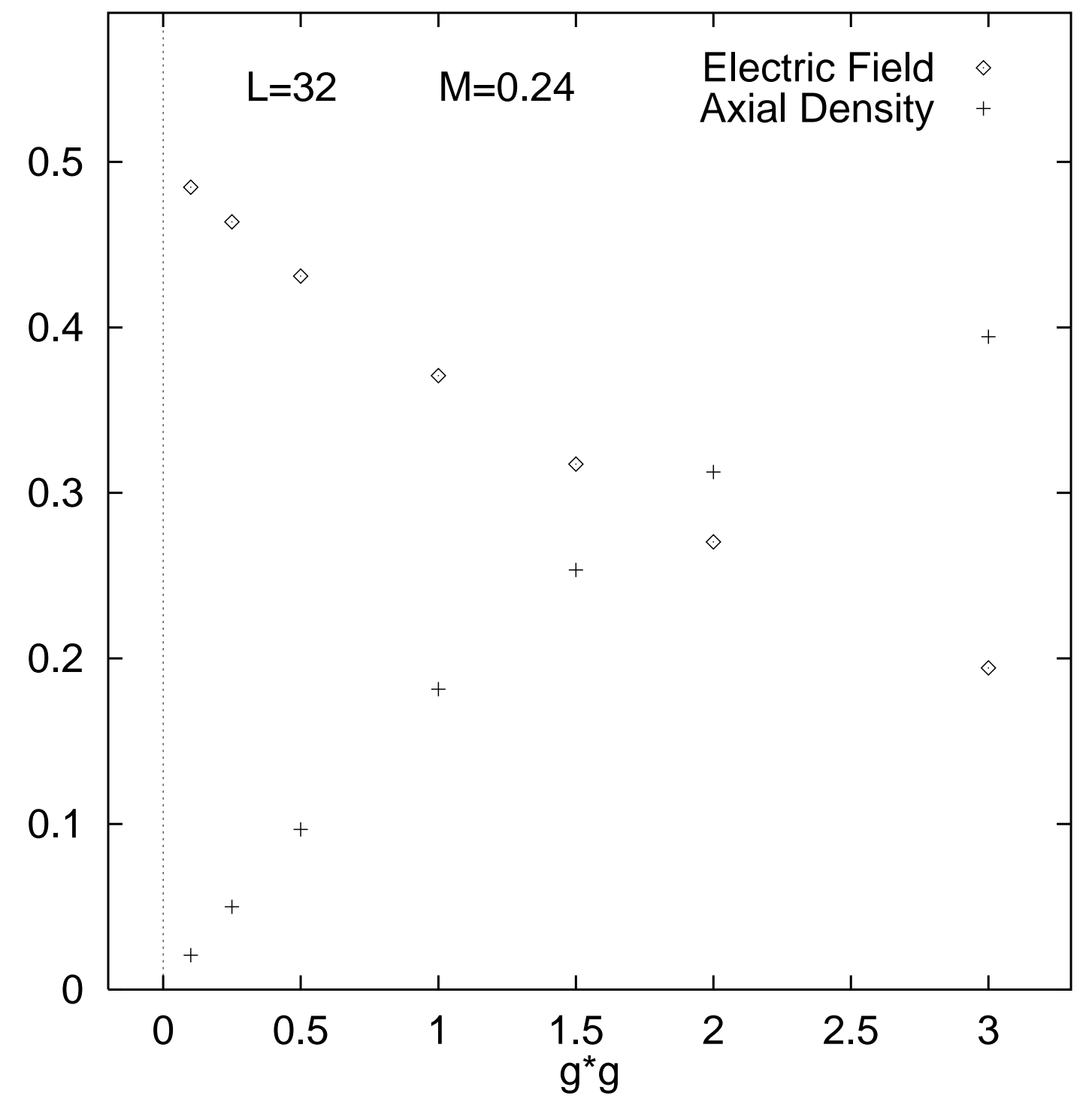

Fig.5 Expectation values of electric field and axial charge density in broken phase. Relative size of the condensates varies with gauge coupling. 


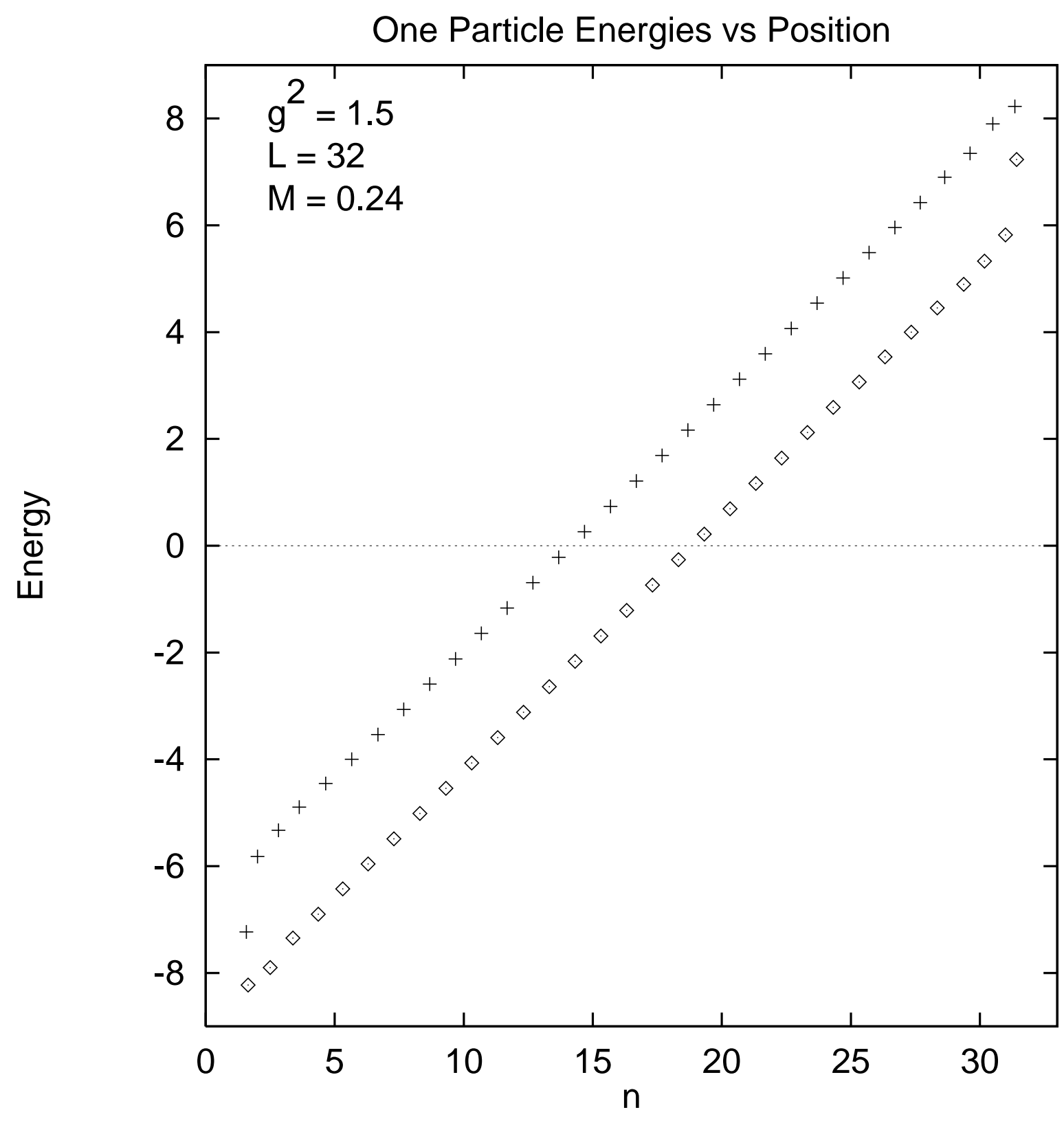

Fig.6 Spatial distribution of the H-F levels in broken phase at $g^{2}=1.5$. Filled levels are marked by the diamonds and empty ones by the crosses. 

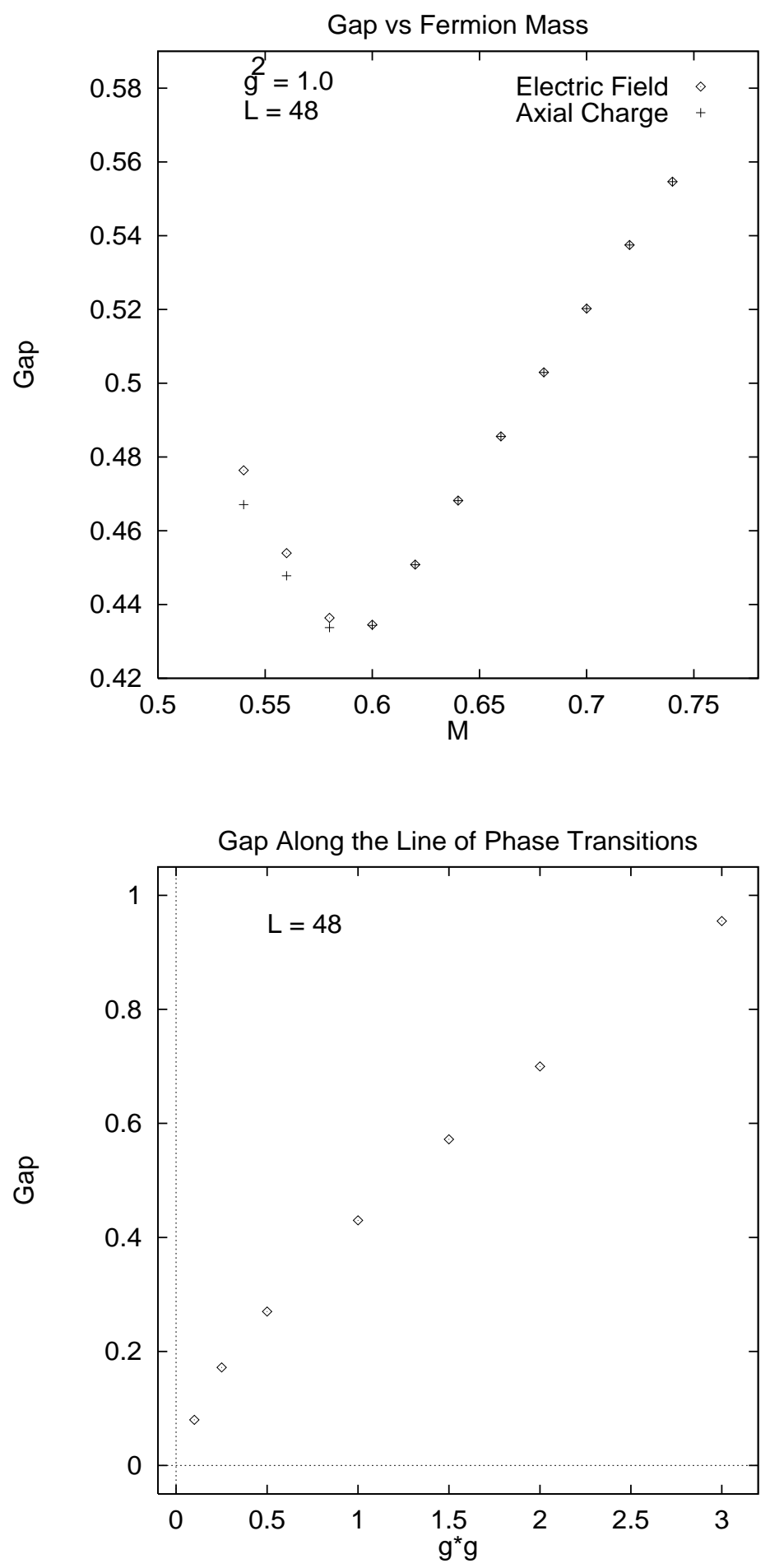

Fig.7a (Upper) Inverse correlation length from electric field and axial charge correlation functions. Fig.7b (Lower) Inverse correlation length of the electric field correlator along the line of phase transitions. 

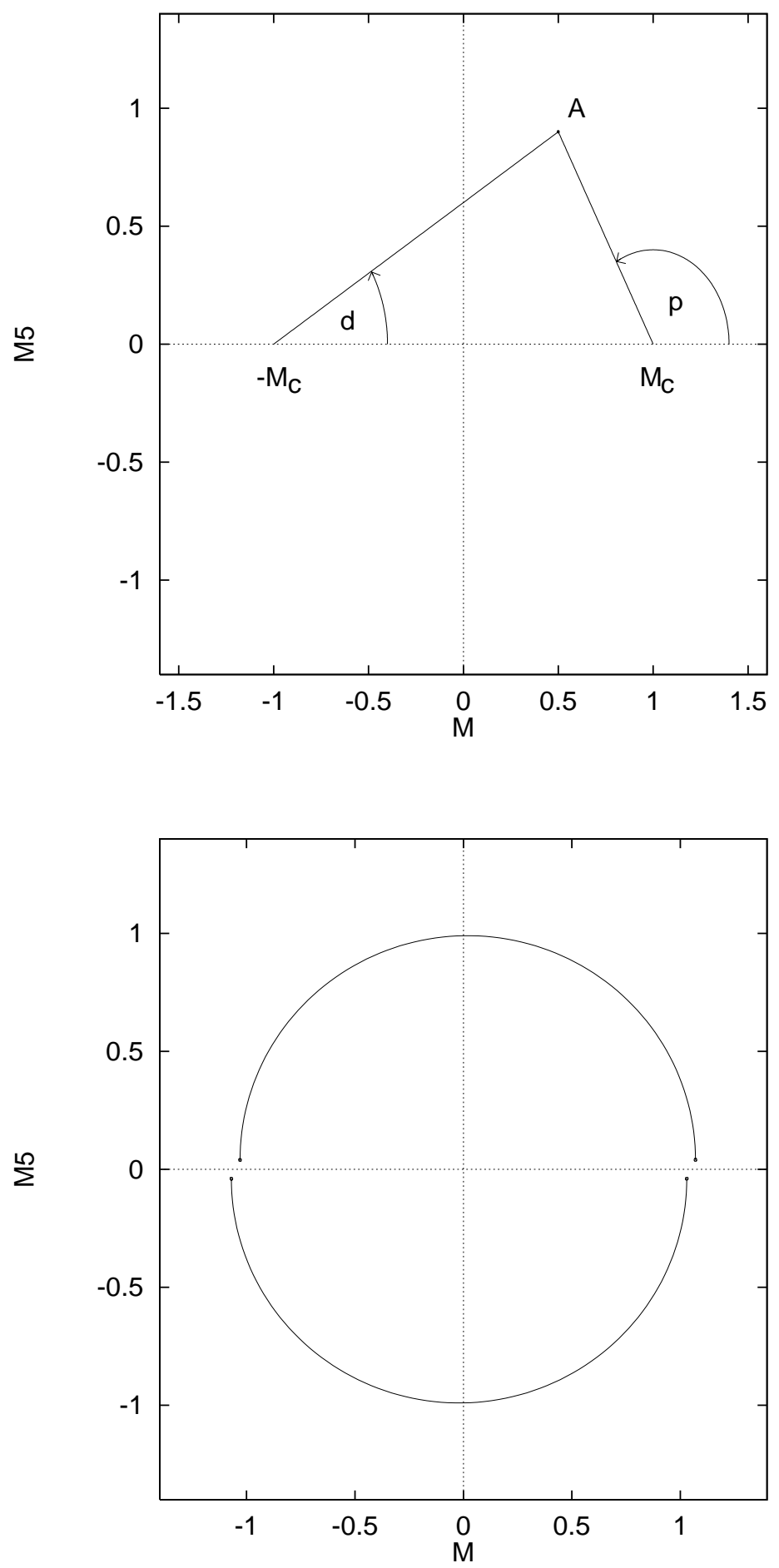

Fig.8a (Upper) Assignements of the angles $\theta_{p}(\mathrm{p})$ and $\theta_{d}(\mathrm{~d})$ of Eq. (41) to a general point $A$ in the $M-M_{5}$ plane. Fig.8b (Lower) Expected phase diagram for two flavours at weak coupling based on the surface mode picture of Ref. [2]. 

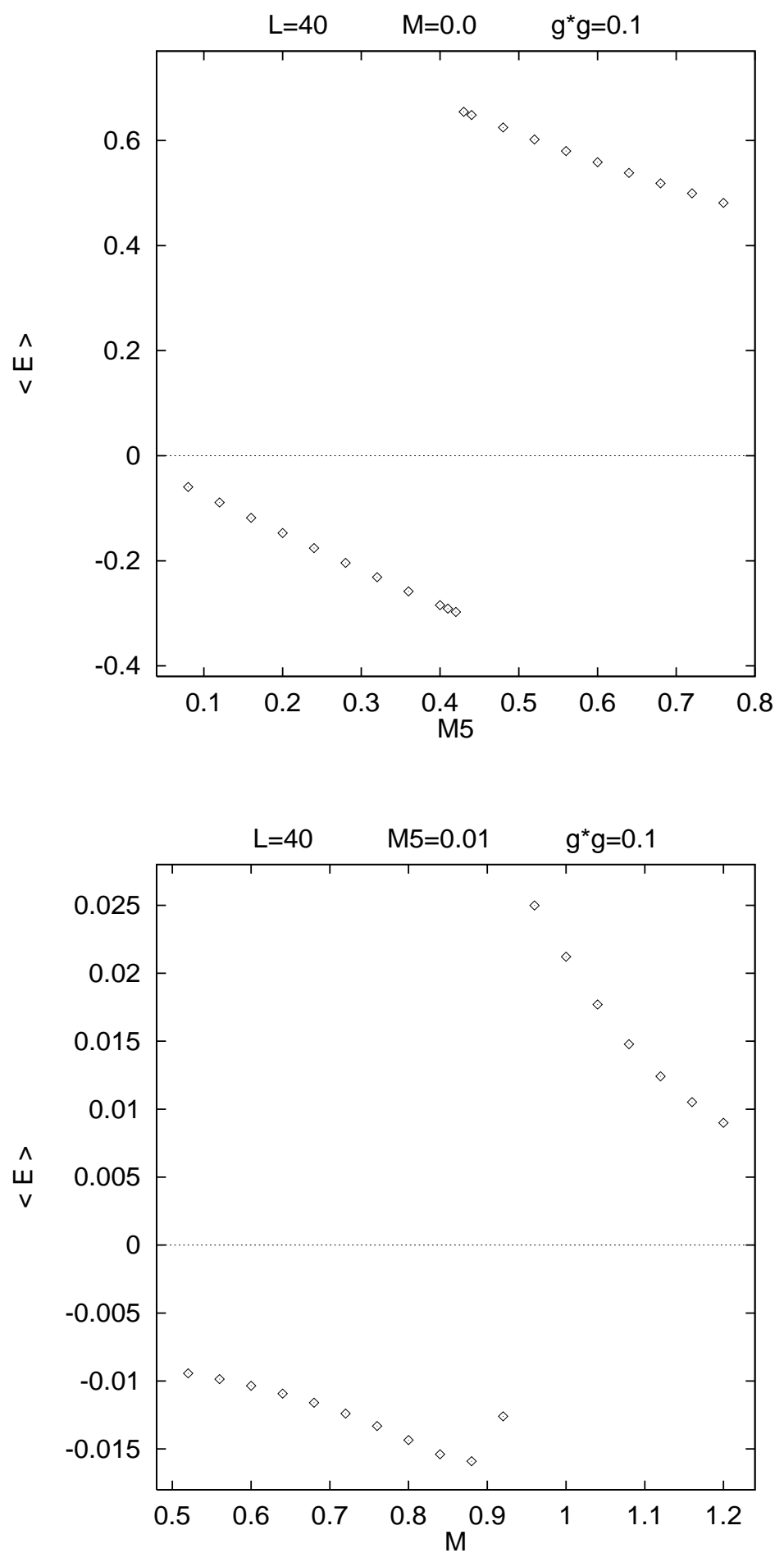

Fig.9a (Upper) Electric field vs $M_{5}$ in the two-flavour model at $g^{2}=0.1$ and $M=0$ on a lattice with 40 sites. Fig.9b (Lower) Electric field along the $M$-axis in the same situation. 


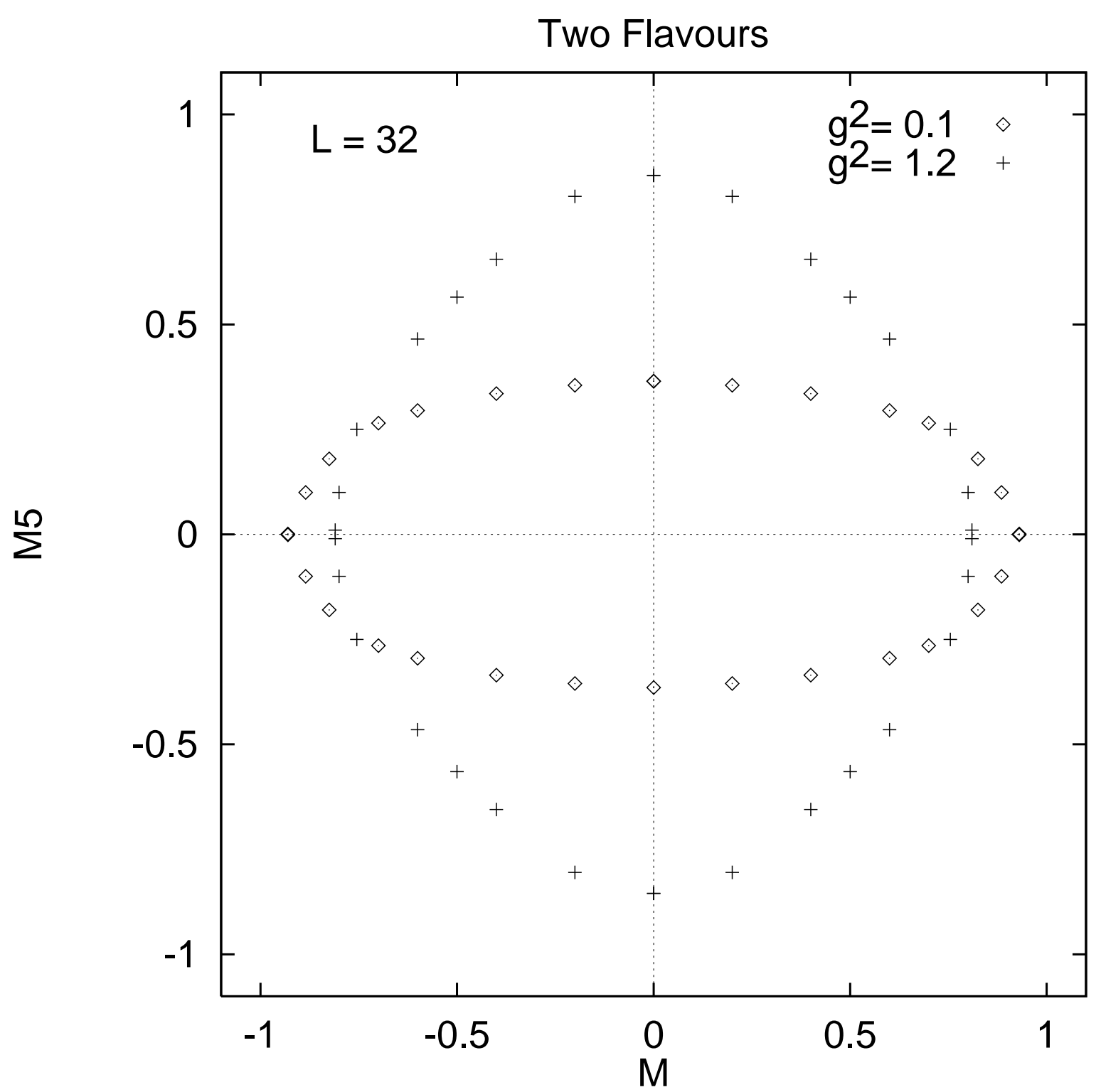

Fig.10 Phase diagrams of the two-flavour model in the $M-M_{5}$ plane at $g^{2}=0.1$ and $g^{2}=1.2$ on a lattice with 32 sites. The points represent the positions where electric field in the Hartree-Fock vacuum changes its sign. 

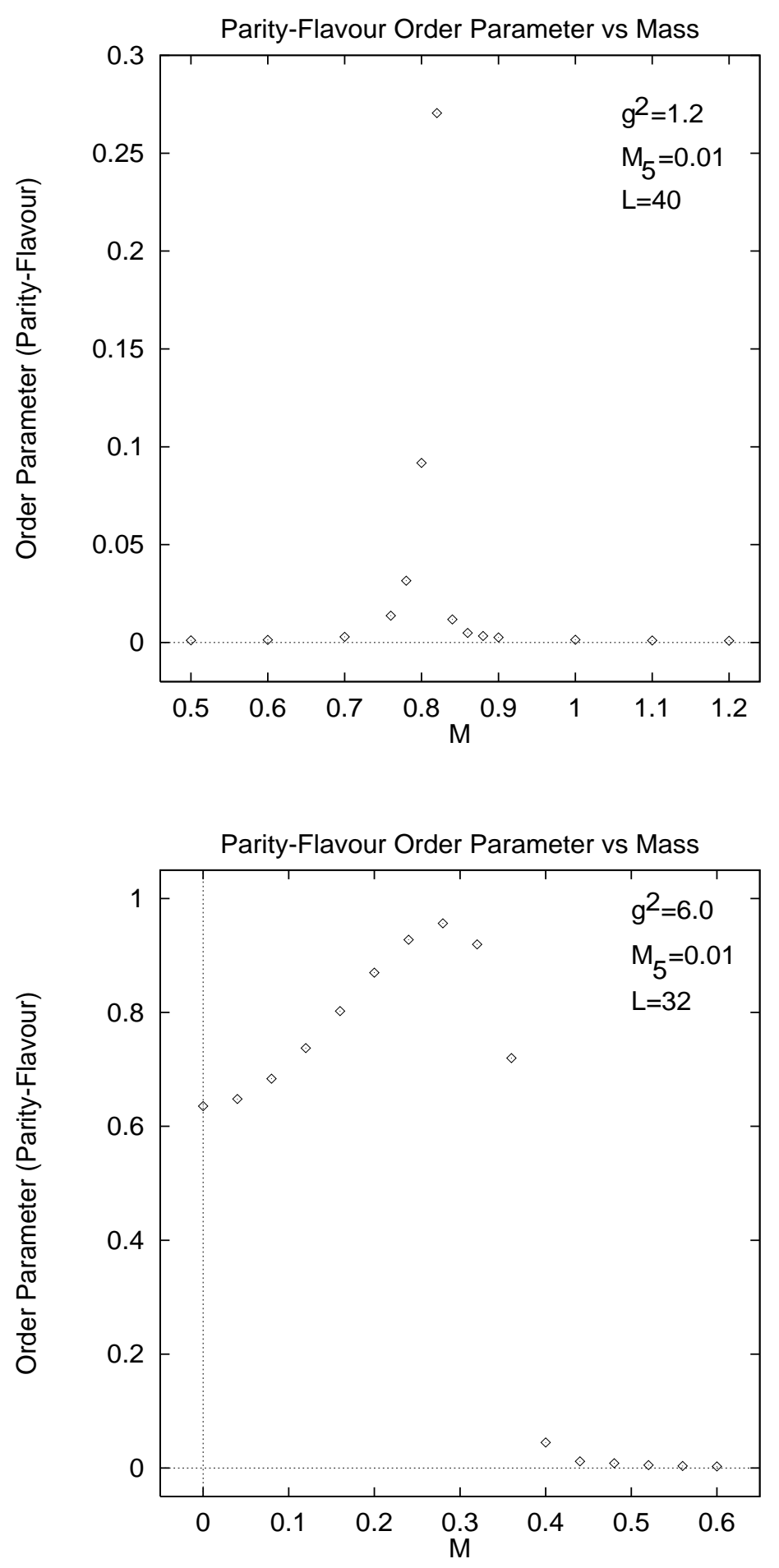

Fig.11a (Upper) Expectation value of $\bar{\psi} \gamma_{5} \tau_{3} \psi$ at $g^{2}=1.2$ and $M_{5}=0.01$ on a lattice with 40 sites. Fig.11b (Lower) The same expectation at $g^{2}=6.0$ on a lattice with 32 sites. 


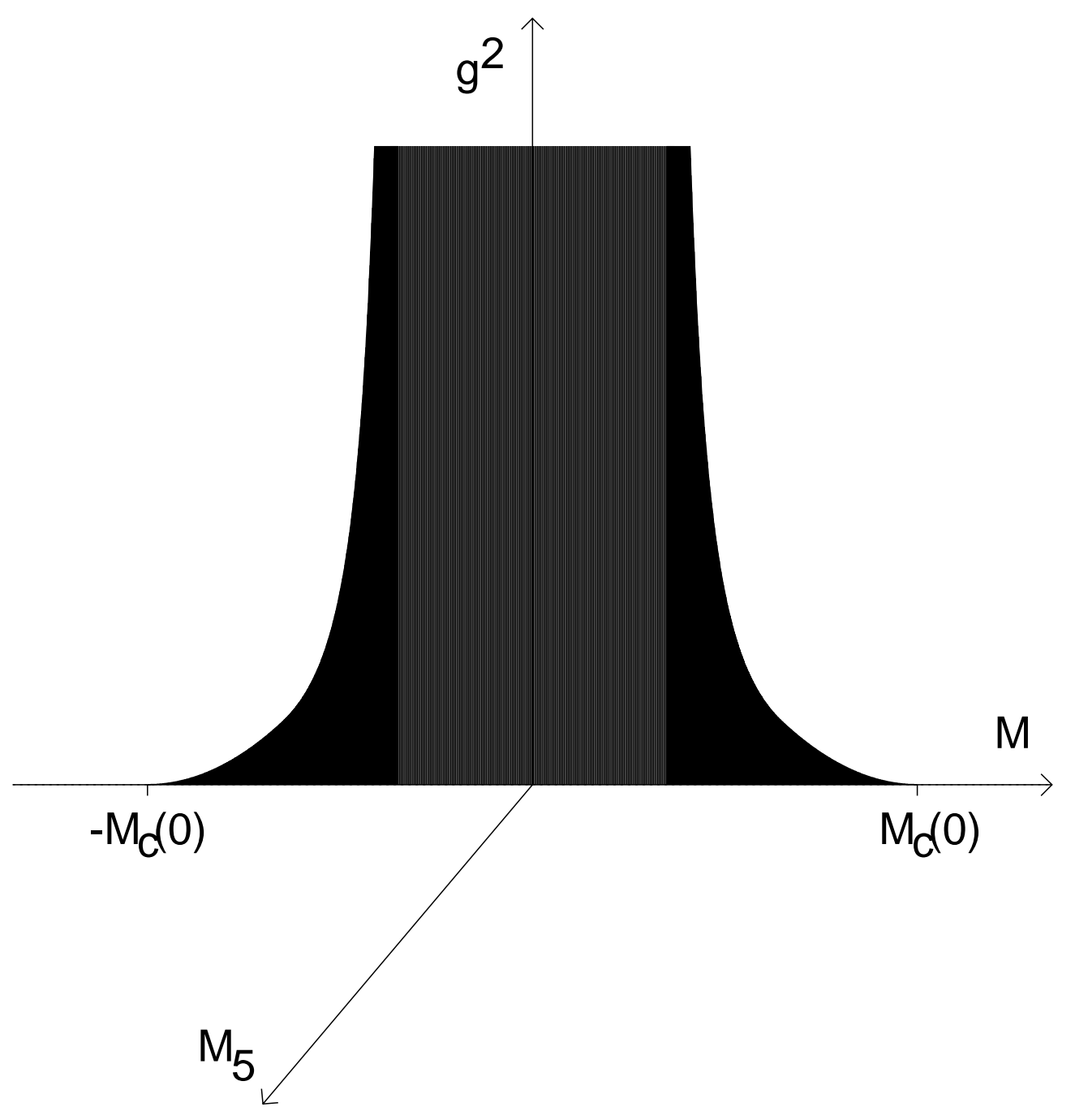

Fig.12 The concluded qualitative behaviour of the full $\mathrm{H}-\mathrm{F}$ phase diagram for the one-flavour Schwinger model on the lattice with Wilson fermions. Parity is spontaneously broken in the black sheet, embedded in the $M-g^{2}$ plane. 

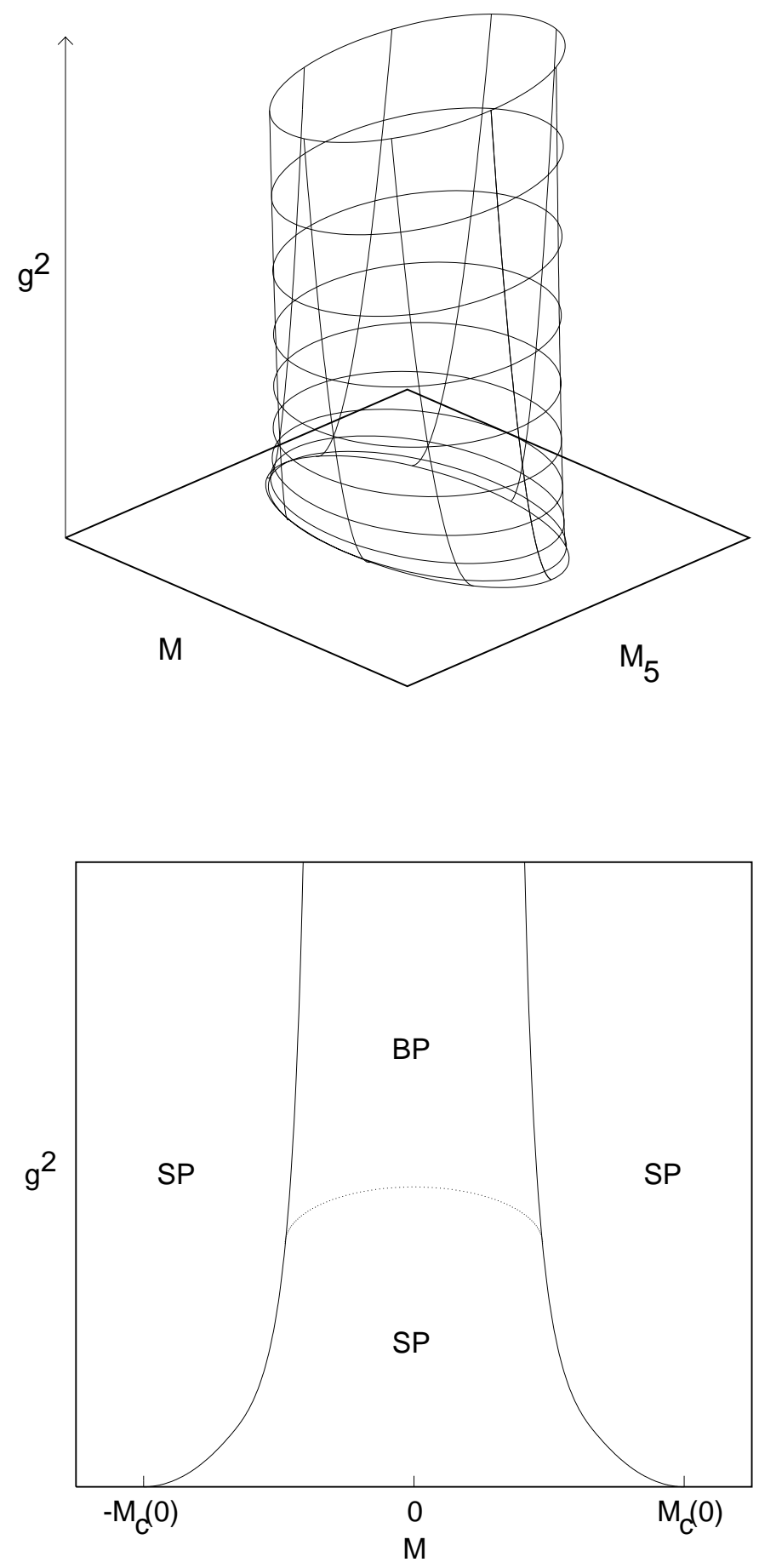

Fig.13a (Upper) The concluded qualitative shape of the surface of " $\theta=\pi$ " transitions for the model with two flavours. Fig.11b (Lower) The H-F phase diagram of this model in $M-g^{2}$ plane. Parity-flavour is spontaneously broken in the "BP" region. 